\title{
Negatively charged Metallacarborane redox couples with both members
}

\section{stable to air.}

\section{Marius Lupu1,§, Adnana Zaulet1,§, Francesc Teixidor1, Eliseo Ruiz2 and Clara}

Viñas1,*

1Institut de Ciència dels Materials, CSIC, Campus UAB, Bellaterra, E-08193, Spain.

${ }^{2}$ Departament de Química Inorgànica and Institut de Recerca de Química Teòrica i Computacional , Universitat de Barcelona, Diagonal 645, E-08028 Barcelona, Spain

e-mail: clara@icmab.es

\begin{abstract}
It was possible to synthesize and isolate redox couples in which both partners are negatively charged. The handy framework is [3,3'-Co(1,2-closo- $\left.\left.\mathrm{C}_{2} \mathrm{~B}_{9} \mathrm{H}_{11}\right)_{2}\right]$. The $\mathrm{E}^{1 / 2}$ potential can be tuned by adjusting the nature and number of substituents on $\mathrm{B}$ and $\mathrm{C}$. The octaiodinated species $\left[3,3^{\prime}-\mathrm{Co}\left(1,2-\text { closo }-\mathrm{C}_{2} \mathrm{~B} 9 \mathrm{H}_{7} \mathrm{I}_{4}\right)_{2}\right]$ - is the most favorable as it is isolatable and stable in air.
\end{abstract}

KEYWORDS: Metallacarboranes, red-ox tuning, red-ox couple, molecular platform, cobaltabisdicarbollide.

$\S$ M. Lupu and A. Zaulet are enrolled in the UAB PhD program. 


\section{Introduction}

The cobaltabisdicarbollide, [3,3'- $\left.\mathrm{Co}\left(1,2-\text { - closo- } \mathrm{C}_{2} \mathrm{~B}_{9} \mathrm{H}_{11}\right)_{2}\right]-$, [1]-[1], is a remarkable anion: it is chemically and thermally stable in a diverse number of situations;[2] it can be substituted at carbon atoms or at boron atoms,[3] and in the latter ones regioselectively at different sites of each one of the two globes.[4] The central core of this anion, " $\mathrm{Co}\left(\mathrm{C}_{2} \mathrm{~B} 3\right)_{2}$ ", is very similar to the core of ferrocene, " $\mathrm{Fe}\left(\mathrm{C}_{5}\right) 2$ ", thus they bear resemblances in some respects, e.g. the reversible electrochemistry[5] and the high chemical and thermal stability,[6] but are different in others, such an enhanced protection of the Co in [1]- by a canopy of boron hydrogen atoms,[7] and properties derived from the six additional atoms placed in two further planes from the core $\left(\mathrm{B}_{5}\right.$ plane and $\left.\mathrm{B}_{1}\right)$. These vertexes produce a rich variety of substitution sites, and are responsible for additional physicochemical properties. One of the most obvious differences between [1]-, and Ferrocene $(F c)$ is the charge of [1]-, that makes the latter and its congeners to be some of the few examples of metallocene type complexes with a negative charge. Previous work has shown that [1]- can be modified by halogenations,[8],[9] and it is a unique framework in its ability to produce a stepwise modulation of its redox potential by each new $\mathrm{B}-\mathrm{X}(\mathrm{X}=$ halogen $)$ unit added.[10] We recently demonstrated that sequential substitution of B-H by B-X units produces an average $\mathrm{E}^{1 / 2}$ shift, near $0.13 \mathrm{~V}$, to more positive potential values.[11] This $\mathrm{E}^{1 / 2}$ cumulative process is very rare[12] and no other redox reversible platform seems to be capable of making it as effectively as [1].. In this work we disclose on i) the $\mathrm{E}^{1 / 2}$ site dependence of two opposite effect substituents, I- vs. Me-, all realized on boron atoms, ii) the power of the $\mathrm{E}^{1 / 2}$ site dependence shift and iii) the application of these concepts to stabilize the $\left[\mathrm{Co}\left(\mathrm{C}_{2} \mathrm{~B}_{9} \mathrm{H}_{11}\right)_{2}\right]_{2}$ framework, $[\mathbf{1}]_{2-}$, in which the $\mathrm{Co}$ is $\mathrm{Co} 2+$. 


\section{Results and Discussion}

Iodine and carbon have similar electronegativities according to the Pauling's scale, $\mathrm{XI}_{\mathrm{I}}=$ 2.66, $\mathrm{X}_{\mathrm{C}}=2.55, \mathrm{X}_{\mathrm{B}}=2.04,[13]$ thus the polarization of the $\sigma$ bonds for $\mathrm{B}-\mathrm{C}$ and $\mathrm{B}-\mathrm{I}$ shall be similar; further, both have the possibility to donate electron density to the cluster cage: iodine by $\pi$-donation and the methyl by hyperconjugation.[14] For both these reasons, we would expect similar effects on $\mathrm{E}_{1 / 2}$ by $\mathrm{B}-\mathrm{C}$ and $\mathrm{B}-\mathrm{I}$ substitution in [1]-; however for $F_{c}$, methyl groups produce an $\mathrm{E}^{1 / 2}$ shift to more negative potentials,[15] whereas bromo groups, taken as similar to iodo, produce an $\mathrm{E}^{1 / 2}$ shift towards more positive potentials, all referenced to pristine $F c$. Therefore, there was the possibility that the opposite trend observed by alkyl or halogen substitution on $F c$ also occurred in [1]-, although its redox couple is $[\mathbf{1}]_{-1 /-2}$ in contrast to $F_{c}$ that is $F_{C+/ 0}$. To provide evidence of this, a set of regioselective derivatives of [1]- were needed. The synthesis of these compounds was now possible due to the great advances in I substitution on the carborane cluster. 4 Earlier we and others had demonstrated that starting from monoiodinated metallacarboranes,[4],[16] alkyl derivatives can be produced by a modification of the Kumada reaction. In Scheme 1, the syntheses of $\left[3,3\right.$ '- $\left.\mathrm{Co}\left(9,12-\mathrm{I}_{2}-1,2-\text { closo- } \mathrm{C}_{2} \mathrm{~B} 9 \mathrm{H}_{9}\right)_{2}\right]-(\mathrm{I} 4-[1]-)$ and $[3,3$ '- $\mathrm{Co}(9,12-(\mathrm{Me}) 2-1,2-$

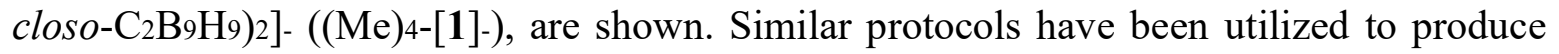
other I- or Me- polysubstituted [1]- derivatives studied in this work.

Chart 1 indicates the numbering of the $\left[3,3^{\prime}-\mathrm{Co}\left(1,2-\right.\right.$ closo- $\left.\left.\mathrm{C}_{2} \mathrm{~B}_{9} \mathrm{H}_{11}\right) 2\right]$ - platform and its corresponding ligand $\left[7,8-\text { nido- } \mathrm{C}_{2} \mathrm{~B}_{9} \mathrm{H}_{11}\right]_{2}$-. In addition to [1] derivatives of the latter have been used, [3,3'-Co(1-Ph-1,2-closo- $\left.\left.\mathrm{C}_{2} \mathrm{~B}_{9} \mathrm{H}_{10}\right) 2\right]-$, [2]-, and [3,3'-Co(1-Me-1,2-closo- $\left.\left.\mathrm{C}_{2} \mathrm{~B}_{9} \mathrm{H}_{10}\right)_{2}\right]-$, [3]- (Figure 1). Derivatives of these frames, the site occupation of the substituent, along with their $E_{1 / 2}^{1 / 2}$ values are in Table 1 while Table 2 is 
dedicated to Me- and I- derivatives of [1]-. Three fundamental aspects stand out: all Mederivatives exhibit an $\mathrm{E}^{1 / 2}$ shift to more negative potential values than the reference value given by [1]-, whereas the $\mathrm{E}_{1 / 2}^{1 / 2}$ for all $\mathrm{I}$ - derivatives are shifted to more positive potential values; second, the power of I- in producing a distinct $\mathrm{E} \frac{1}{2}$ shift is far superior to the methyl groups, and third an $\mathrm{E}_{1 / 2}^{1 / 2}$ substituent site dependence is clear. Therefore, B-Me or B-I substitution on [1]- produce opposite effects on E1/2 just as $\mathrm{C}-\mathrm{Me}$ and $\mathrm{C}-\mathrm{Br}$ in $F c$.

The stepwise substitution of B-H by B-Me induces shifts of $\mathrm{E}_{1 / 2}^{1 / 2}$ to more negative potential values that are very small, between $-10 /-15 \mathrm{mV}$ each, thus one substitution produces -10 $\mathrm{mV}$ shift; two substitutions produce $-20 \mathrm{mV}$; four, $-40 \mathrm{mV}$; six, $-90 \mathrm{mV}$; eight, $-120 \mathrm{mV}$. The shift induced by each methyl unit in [1]- is about $1 / 4$ of the effected on $F c$. Further, these changes are so small that they cannot be used to define a site effect.

Much more relevant on $\mathrm{E}_{1 / 2}^{1 / 2}$ are the stepwise substitutions of $\mathrm{B}-\mathrm{H}$ by B-I. To interpret the results, it is advisable to formally slice each half of [1]- so that four planes are generated. First plane is made of $\mathrm{Co}$; second plane is made of $\mathrm{B}(4,7,8)$ and $\mathrm{C}(1,2)$; third plane is made of $\mathrm{B}(5,6,9,11,12)$; and fourth plane is made by $\mathrm{B}(10)$. The site influence on the $\mathrm{Co} 3+/ 2+$ couple can be studied by computing the influence of each substituent on each plane on $\mathrm{E}^{1 / 2}$. The B-I's on $\mathrm{B}(4,7,8)$ will tell on the effect of the substituent on the plane $\mathrm{C}_{2} \mathrm{~B}_{3}$ bonded to Co. From Table 2 entry 7, I-B(8) exerts a $\Delta \mathrm{E}_{1 / 2}^{1 / 2}$ near $+0.30 \mathrm{~V}$; the existence of a second I-B in the equivalent position $\mathrm{B}\left(8^{\prime}\right), \mathrm{I}_{2}-[\mathbf{1}]$ - (Table 2 entry 9 ), induces an average $\Delta \mathrm{E}^{1 / 2}$ per iodine atom, near $+0.24 \mathrm{~V}$; the influence of the equivalent $\mathrm{B}(4)$ and $\mathrm{B}(7)$ sites can be obtained from I4-[1]- in Table 2 entry 12 yielding an average of $+0.26 \mathrm{~V}$, or from I2-[1]- (Table 2, entry 10) with an average of $+0.28 \mathrm{~V}$. Thus, it could be assumed that each B-I on the nearest plane to the Co, produces a $\Delta \mathrm{E}^{1 / 2}$ of $+0.27 \pm 0.03 \mathrm{~V}$. A similar process can be followed for the third plane $\mathrm{B} 5$, the second nearest to $\mathrm{Co}$. In this case, each B-I produces a $\Delta \mathrm{E}^{1 / 2}$ of $0.15 \pm 0.03 \mathrm{~V}$. 
Finally, a B-I on the fourth plane produces a $\Delta \mathrm{E}^{1 / 2}$ of $+0.14 \mathrm{~V}$. Noticeably, these results are related to the B-I vertex distance to the Co metal center. If the rough approximation of the front view of the icosahedron cluster to a hexagon is made, as shown in Figure 2, and the Co is made to occupy one of the vertexes, the distance to the substituted boron in the second plane is $\boldsymbol{d}$, to the third one is $1.73 \boldsymbol{d}$, and to the fourth one is $2 \boldsymbol{d}$. If $\boldsymbol{d}$ is taken as 0.27 , according to the influence of a B-I in the nearest plane to Co, $d / 1.73$ is 0.16 and $d / 2$ is 0.14 that nicely fits with the $\Delta \mathrm{E}^{1 / 2}$ values experimentally obtained, $0.27 \pm 0.03 \mathrm{~V}, 0.15 \pm 0.03 \mathrm{~V}$ and $0.14 \mathrm{~V}$.

The former values validate the additive rule and enlighten the importance of the site where the substitution occurs. These site dependent $\Delta \mathrm{E}_{1 / 2}$ values indicate that a hypothetical [1]derivative with $6 \mathrm{~B}-\mathrm{I}$ in the two planes nearest to Co, would induce a global $\Delta \mathrm{E}^{1 / 2}$ near $+1.62 \mathrm{~V}$, thus converting [1]- with $\mathrm{E}_{1 / 2}^{1 / 2}=-1.80 \mathrm{~V}$ into $\mathrm{I} 6-[1]$ - with $\mathrm{E}_{1 / 2}^{1 / 2}$ near $-0.18 \mathrm{~V}$. Such a synthesis is still out of the possibility of current state of the art in halogen substitution but a good approach is I4-[1]- (Table 2, entry 12) that with $50 \%$ of the B-H replaced in I8-[1](Table 2, entry 15) almost reaches the same global $\Delta \mathrm{E}^{1 / 2}$ as the latter. As a proof of concept, the anion I6-[1]- B(4,4',9,9',12,12') was synthesized and its expected $\mathrm{E}^{1 / 2}$ should be near $0.66 \mathrm{~V}$. The experimental $\mathrm{E}^{1 / 2}$ value is $-0.80 \mathrm{~V}$.

Recently, [17] based on seminal works of Pombeiro,[18] Pickett,[19] Lever[20] and Bursten[21] groups, reviewed on the importance of establishing a relationship between the redox potential-structure of complexes that define electrochemical parameters, which are shown for a wide variety of ligands and metal sites. Lever and co-workers reported,[20] an electrochemical parametrization in sandwich complexes of the first row transition metals can be established by assigning electrochemical parameters to the ligands.[22] Due to the sandwich nature of the cobaltabisdicarbollide anions, we adhered recently to Lever's 
approach[23] to calculate from the experimental $E_{1 / 2}$ value the $E_{L}$ contribution of the ligand [7,8- $\left.\mathrm{C}_{2} \mathrm{~B}_{9} \mathrm{H}_{11}\right]_{2}-$ taken for $\left[3,3^{\prime} \mathrm{Co}-\left(1,2-\mathrm{C}_{2} \mathrm{~B}_{9} \mathrm{H}_{11}\right)_{2}\right]$ - and two diansa metallacyclophane derivatives, phospha[1]cobaltabisdicarbollidephane and benzene[2]cobaltabisdicarbollidephane. The estimated values were $-0.00(0.10),+0.22$ and $0.35 \mathrm{~V}$, respectively.[23] The fact that cobaltabisdicarbollide followed Lever's equation derived mostly from metallocenes, indicated that cobaltabisdicarbollide is a metallocenelike molecule that behaves as such.[23] This is why, following the same procedure, $E_{L}$ contribution of the different methylated and iodinated $\left[7,8-\mathrm{C}_{2} \mathrm{~B}_{9} \mathrm{H}_{11}\right] 2$ - ligand derivatives have been calculated in this work. The $E_{L}$ ligands' contributions are displayed in Table 2 . The stepwise $\Delta \mathrm{E}^{1 / 2}$ alteration was correlated with theoretical calculations.[24] Free energy calculations were performed for the three optimized isomers studied (see Figure 3) considering the fifteen $\mathrm{Co} 2+$ and $\mathrm{Co}_{3}+$ complexes $1-15$. The calculated stability shows that the $\mathrm{c}$ isomer is usually the most stable one (see Table 3), only for the $\mathbf{1 2}$ complexes, the simultaneous $4,4^{\prime}, 7,7^{\prime}$ substitution destabilizes such isomer. The substitution of the hydrogen atoms by Me- groups (entries 1-5 in Table 2) has a small influence in the redox potential due to their low participation in the HOMO orbital (Figure 4).

However, in the case of I8-[1]- (see 15 in Figure 4) the HOMO energy is considerably stabilized and consequently increases the energy required for the oxidation process (less negative redox potentials). It is worth noting the almost perfect linear correlation between the calculated DFT redox potentials and the calculated HOMO energies for the solvated molecules (Figure 4). Such correction is valuable as the HOMO energies are easily calculated without performing calculations of the vibrations mandatory to estimate thermodynamic magnitudes, such entropy or free energy. As we have been claiming 
throughout the paper, based on experimental evidence, the computational studies also indicate strong $\mathrm{E}_{1 / 2}^{1 / 2}$ dependence on the site of the substituent. As it can be seen in Figure 4 for $\mathbf{1 5}$, the iodine orbital contribution is larger at the 8 and 10 positions $(2$ nd and 4 rt planes in Figure 2) than in 9 and 12 (3rd plane). Hence, the existence of substituent in the 2 nd and 4 th planes should be more efficient in terms of $E^{1 / 2}$ tuning than substituent on the 3 rd plane. This is in agreement with the values in Table 2 and with Figure 2 for the 2 nd and 3rd planes. The mismatch with the 4th plane may have been due to a certain degree of shadowing of the $\mathrm{E}^{1 / 2}$ tuning at position 10 caused by the substituent on the 3rd level.

It is clear from Table 2 that I8-[1]- is the [1]- derivative with the highest positive $\mathrm{E}_{1 / 2}^{1 / 2}$ value. Further, it undergoes a perfect one-electron reversible redox process. The redox potential of I8-[1]-/I8-[1] 2- pair is $-0.68 \mathrm{~V} v s . F C+/ F c$.

Conversely, [1] - has a very negative $\mathrm{E}^{1 / 2}$ value, $-1.80 v_{s} F_{C+} / F_{c}$. The results shown here for I8-[1] - indicate a strategy to lower, making more positive, the $E_{1 / 2}^{1 / 2}$ value of the platform [1]to produce the uncommon $-1 /-2$ reversible couples. Moreover, we wanted to learn on the stability of both members of the uncommon redox couple $[\mathbf{1}]-1 /-2$ with a practical $E_{1 / 2}^{1 / 2}$ value. In [1]-, the Co is $\mathrm{Co3}^{+}$, and as the dicarbollide is a high field ligand, the 6 electrons $\mathrm{d}$ are paired, thus [1]- shall be a diamagnetic species. In [1]2-, there are 7 electrons $\mathrm{d}$, and a paramagnetic species is expected. For [1]-, the independent oxidized and reduced species would be difficult to be observed, indeed the reduced form has never been reported nor isolated, but in I8-[1]- the chances to observe both redox partners should be much higher. Furthermore, tests on the stability of both forms, oxidized and reduced, in not highly strict anaerobic conditions would be a good indication of possible applications of these complexes. In a typical reaction the $\left[\mathrm{NMe}_{4}\right]+$ salt of I8-[1]- was dissolved in THF and mixed with freshly prepared $\mathrm{Na}\left[\mathrm{C}_{10} \mathrm{H}_{8}\right]$. Immediately the color of the solution turned from light 
orange to dark red. Progress of the reaction was followed by NMR analysis directly from the crude and the ${ }_{11} \mathrm{~B}\{1 \mathrm{H}\}-\mathrm{NMR}$ spectrum gave four well defined NMR resonances with intensities 2:6:4:6 in a wide field range $(+28 /-95 \mathrm{ppm})$, clearly suggesting that the generated species was paramagnetic. Following oxidation by air, the sample returned to the original $\mathrm{Co}_{3}+$ color and its $11 \mathrm{~B}\{1 \mathrm{H}\}-\mathrm{NMR}$ spectrum to the expected range $(-2 /-20 \mathrm{ppm})$. Both ${ }_{11} \mathrm{~B}\{1 \mathrm{H}\}-\mathrm{NMR}$ spectra for I8-[1] - (in red) and I8-[1] 2 - (in black) are shown in Figure 5. The ${ }_{11} \mathrm{~B}\{1 \mathrm{H}\}-\mathrm{NMR}$ for $\mathrm{I} 8-[\mathbf{1}]$ - evidences the diamagnetic nature of the compound. Conversely the $11 \mathrm{~B}-\mathrm{NMR}$ of I8-[1] 2- expands significantly evidencing the paramagnetic nature of the reduced form. This is the first reported ${ }_{11} \mathrm{~B}\{1 \mathrm{H}\}-\mathrm{NMR}$ spectrum of one derivative of the cobaltabisdicarbollide platform proving the stability of the reduced species. To detect the presence of $\mathrm{Co}_{2+}$, the EPR spectrum of a $33 \mathrm{mM}$ solution of $\left.\mathrm{I}_{8-}[\mathbf{1}]\right]_{2}$ in $\mathrm{H}_{2} \mathrm{O}$ was recorded at $130 \mathrm{~K}$ showing a $\mathrm{g}=2,021$ (Figure 6). With these results in hand other tests were done to isolate I8-[1]2-.

To this objective, the crude of the reaction between $\mathrm{I}_{8}-[\mathbf{1}]$ - and $\mathrm{Na}\left[\mathrm{C}_{10} \mathrm{H}_{8}\right]$ was filtered to remove the existing solid whose IR did not display any B-H absorption. The solvent was evaporated and the naphthalene was recovered by low pressure sublimation. The final product was collected as a dark brown solid and the further ${ }_{11} \mathrm{~B}\{1 \mathrm{H}\}-\mathrm{NMR}$ analysis showed that it was not altered during the purification process. Moreover, the stability in both air and inert gas $\left(\mathrm{N}_{2}\right.$ and Ar) conditions was also checked. The I8-[1]2- species in solid state was perfectly air-stable for 7 days, while under inert conditions its stability increased to more than 1 month (Figure 7).

The THF solution of the reduced I8-[1]2- species was stable for several hours in air. After one day, some re-oxidation was observed. 
Stable paramagnetic compounds derivatives of neutral $\mathrm{C}_{2} \mathrm{~B}_{10}$ and anionic [CB11]- clusters have been recently reported[25] but this reduced I8-[1]2- species is the first isolated and stable paramagnetic cobaltabisdicarbollide, which is the most commonly used and widely studied metallacarborane.

\section{Conclusions}

The wide pattern of possible substitutions in [3,3'- $\mathrm{Co}\left(1,2\right.$-closo- $\left.\left.\mathrm{C}_{2} \mathrm{~B}_{9} \mathrm{H}_{11}\right) 2\right]$ - has facilitated the preparation of a set of regioselective polyiodinated derivatives, which in turn, by a Kumada inspired B-C cross coupling, has led to several regioselective polymethylated derivatives. A singular property of $\left[3,3^{\prime}-\mathrm{Co}\left(1,2-\operatorname{closo}-\mathrm{C}_{2} \mathrm{~B}_{9} \mathrm{H}_{11}\right)_{2}\right]$ - is that it is redox reversible and permits tailoring the redox potential very accurately. No other platform allows for such an adjustment. Boron dehydromethylation shifts the $\mathrm{E}_{1 / 2}^{1 / 2}$ value to more negative potential values, whereas boron dehydroiodination does the opposite effect. The redox potential shift can be large, even larger than $1 \mathrm{~V}$, because of the cumulative individual effects, that are also site dependent. The effect of the B-I unit on the $\mathrm{E}^{1 / 2}$ value is inversely proportional to the distance. The air exposure of $\left[3,3^{\prime}-\mathrm{Co}\left(1,2-\text { closo- } \mathrm{C}_{2} \mathrm{~B}_{9} \mathrm{H}_{11}\right)_{2}\right]_{2}$ is totally impractical, however from the $\mathrm{E} / 2$ shift to more positive potentials of the couple $\left[3,3^{\prime}-\mathrm{Co}\left(8,9,10,12-\mathrm{I}_{4}-1,2-\text { closo }-\mathrm{C}_{2} \mathrm{~B} 9 \mathrm{H} 7\right)_{2}\right]-1 /-2$ caused by 8 dehydroiodinations, the $\mathrm{E}_{1 / 2}$ has shifted from -1.80 to $-0.68 \mathrm{~V} v s F_{c}+F_{c}$. This $\mathrm{E}^{1 / 2}$ shift allows both the oxidized and reduced forms of the couple $\left[3,3^{\prime}-\mathrm{Co}\left(8,9,10,12-\mathrm{I} 4-1,2-\right.\right.$ closo $\left.\left.-\mathrm{C}_{2} \mathrm{~B} 9 \mathrm{H} 7\right) 2\right]-1 /-2$ standing in air for several hours and days. These results open an unexplored way to adjust the desired $\mathrm{E}_{1 / 2}$ value in devices in which $\mathrm{E}_{1 / 2}^{1 / 2}$ modification will not imply a sharp molecular modification. In this paper, the redox potential of a series of related complex of $\mathrm{Co}_{3}+$ has allowed for the 
quantification of the ligands electron acceptor/donor properties, which can then be applied as an important characterization parameter.

\section{Experimental section}

\section{Instrumentation.}

IR spectra ( $v, \mathrm{~cm}-1 ;$ ATR or $\mathrm{KBr}$ pellets) were obtained on a Shimadzu FTIR-8300 spectrophotometer. The ${ }_{1} \mathrm{H}-$ and ${ }_{1} \mathrm{H}\left\{{ }_{11} \mathrm{~B}\right\}-\mathrm{NMR}(300.13 \mathrm{MHz}),{ }_{13} \mathrm{C}\left\{{ }_{1} \mathrm{H}\right\}-\mathrm{NMR}(75.47$ $\mathrm{MHz})$ and ${ }_{11} \mathrm{~B}-$ and ${ }_{11} \mathrm{~B}\{1 \mathrm{H}\}-\mathrm{NMR}(96.29 \mathrm{MHz})$ spectra were recorded on a Bruker ARX 300 instrument equipped with the appropriate decoupling accessories. All NMR spectra were performed in acetone deuterated solvent at $22^{\circ} \mathrm{C}$. The ${ }_{11} \mathrm{~B}-$ and ${ }_{11} \mathrm{~B}\{1 \mathrm{H}\}-\mathrm{NMR}$ shifts were referenced to external $\mathrm{BF}_{3} \cdot \mathrm{OEt} 2$, while the ${ }_{1} \mathrm{H},{ }_{1} \mathrm{H}\left\{{ }_{11} \mathrm{~B}\right\}$ and ${ }_{13} \mathrm{C}\{1 \mathrm{H}\}-\mathrm{NMR}$ shifts were referenced to SiMe4. Chemical shifts are reported in units of parts per million downfield from reference, and all coupling constants in Hz. The mass spectra were recorded in the negative ion mode using a BrukerBiflex MALDI-TOF-MS $\left[\mathrm{N}_{2}\right.$ laser; $\lambda$ exc $337 \mathrm{~nm}$ (0.5 ns pulses); voltage ion source $20.00 \mathrm{kV}$ (Uis1) and $17.50 \mathrm{kV}$ (Uis2)].

For voltammetric determinations, an electrochemical system, VoltaLab (Universal Electrochemical Laboratory System) interfaced with a PGZ100 potentiostat (Radiometer Analytical) and controlled by the VoltaMaster 4 software, was used. The electrochemical cell contained glassy carbon electrode as working electrode, a reference $\mathrm{Ag} / \mathrm{AgCl} / \mathrm{KCl}$ sat electrode and platinum wire as auxiliary electrode. The solutions were deaerated with analytical grade nitrogen at the start of each experiment to prevent oxygen interference. All experiments were performed at room temperature. Cyclic voltammogram responses were recorded at glassy carbon electrode in $\mathrm{MeCN}$ of $5 \cdot 10_{-3} \mathrm{M} \ln _{-}[1]-$ using $[\mathrm{NBu} 4]\left[\mathrm{PF}_{6}\right](0.1 \mathrm{M})$ as supporting electrolyte. All the potential values were referred to the $F_{c+} / F_{c}$ couple $\left[\mathrm{E}_{1 / 2}\right.$ 
$(F c+/ F c)=0.64 \mathrm{~V} v s$. Standard Hydrogen Electrode (SHE)].

Materials. Unless otherwise noted, all metallacarborane anions prepared are air and moisture stable. All manipulations were carried out under inert atmosphere. 1,2dimethoxyethane (DME) and THF were distilled from sodium benzophenone prior to use. Reagents were obtained commercially and used as purchased. 1,2-closo- $\mathrm{C}_{2} \mathrm{~B}_{10} \mathrm{H}_{12}$ was obtained from Katchem. 1-Me-1,2-closo- $\mathrm{C}_{2} \mathrm{~B}_{10} \mathrm{H}_{11,[26]}$ 1-Ph-1,2-closo- $\mathrm{C}_{2} \mathrm{~B}_{10} \mathrm{H}_{11,[27]}$ [9-I1,2-closo- $\left.\mathrm{C}_{2} \mathrm{~B}_{10} \mathrm{H}_{11}\right]_{,[28]}$ [9-I-7,8-nido- $\left.\mathrm{C}_{2} \mathrm{~B} 9 \mathrm{H}_{11}\right],[29]$ [9,11-I2-7,8-nido- $\left.\mathrm{C}_{2} \mathrm{~B}_{9} \mathrm{H}_{10}\right],[30]$ [5,6-I27,8-nido- $\left.\mathrm{C}_{2} \mathrm{~B} 9 \mathrm{H}_{10}\right]-,[11] \quad\left[1,5,6,10-\mathrm{I} 4-7,8-\right.$ nido- $\left.\mathrm{C}_{2} \mathrm{~B} 9 \mathrm{H} 8\right]-,[11] \quad[3,3$ '-Co-(9,12-I2-1,2-closo$\left.\left.\mathrm{C}_{2} \mathrm{~B} 9 \mathrm{H} 9\right)_{2}\right]-,[11] \quad\left[3,3\right.$ '-Co- $\left.\left(8,9,10,12-\mathrm{I} 4-1,2-\text { closo- } \mathrm{C}_{2} \mathrm{~B} 9 \mathrm{H} 7\right)_{2}\right]-,[11] \quad\left[3,3^{\prime}-\mathrm{Co}(8-\mathrm{I}-1,2-\right.$ closo$\left.\mathrm{C}_{2} \mathrm{~B}_{9} \mathrm{H}_{10}\right)\left(1^{\prime}, 2^{\prime}\right.$-closo- $\left.\left.\mathrm{C}_{2} \mathrm{~B}_{9} \mathrm{H}_{11}\right)\right]-,[31] \quad\left[3,3^{\prime}-\mathrm{Co}\left(8-\mathrm{I}-1,2-\text { closo-C} 2 \mathrm{~B}_{9} \mathrm{H}_{10}\right)_{2}\right]-,[11],[31] \quad[3,3$ '-Co(8Me-1,2-closo- $\left.\mathrm{C}_{2} \mathrm{~B}_{9} \mathrm{H}_{10}\right)\left(1^{\prime}, 2^{\prime}\right.$-closo- $\left.\left.\mathrm{C}_{2} \mathrm{~B}_{9} \mathrm{H}_{11}\right)\right]-,[31]$ [3,3'-Co-(8-Me-1,2-closo- $\left.\left.\mathrm{C}_{2} \mathrm{~B}_{9} \mathrm{H}_{9}\right) 2\right]-,[31]$ 3,3'-Co(8,9,10-I3-1,2-closo- $\left.\left.\mathrm{C}_{2} \mathrm{~B} 9 \mathrm{H} 8\right)_{2}\right]-,[16], 3$ '-Co(8,9,10-Me3-1,2-closo- $\left.\left.{ }_{2} \mathrm{~B} 9 \mathrm{H} 8\right)_{2}\right]-,[16]$ were synthesized as reported in the literature. The synthesis of [3,3'-Co-(8-Me-1,2-closo$\left.\left.\mathrm{C}_{2} \mathrm{~B} 9 \mathrm{H}_{9}\right)_{2}\right]$ - and [3,3'-Co-(9,12-I2-1,2-closo- $\left.\left.\mathrm{C}_{2} \mathrm{~B} 9 \mathrm{H}_{9}\right)_{2}\right]$ - has been also reported by using a different method.[8]

\section{Synthesis of [HNMe3][5-I-7,8-nido-C2 $\left.\mathrm{B}_{9} \mathrm{H}_{11}\right]$}

To a solution of $\mathrm{KOH}(418 \mathrm{mg}, 7.46 \mathrm{mmol})$ in degassed EtOH $(10 \mathrm{ml})$, 9-I-1,2-closo$\mathrm{C}_{2} \mathrm{~B}_{10} \mathrm{H}_{11}(252 \mathrm{mg}, 0.93 \mathrm{mmol}$ ) was added. The solution was refluxed for $5 \mathrm{~h}$. After cooling down to room temperature, the solvent was removed under reduced pressure and the solid residue was dissolved in $10 \mathrm{ml}$ of water. The solution was neutralized with $\mathrm{HCl} 1 \mathrm{M}$. Afterwards, aqueous solution of $\left[\mathrm{HNMe}_{3}\right] \mathrm{Cl}$ was added dropwise until no more precipitate was formed. The white solid was filtered and rinsed with water to give [HNMe3][5-I-7,8nido- $\left.\mathrm{C}_{2} \mathrm{~B}_{9} \mathrm{H}_{11}\right]$ (250 mg, 83\%). Elemental analysis for $\mathrm{C}_{5} \mathrm{H}_{21} \mathrm{~B} 9 \mathrm{IN}$ : calc: C 18.80, H 6.63, N 4.38; found C 18.39, H 6.31, N 4.27. IR (KBr): v=3020 (s, vs $\left(\mathrm{C}_{\mathrm{c}-\mathrm{H}}\right), 2947,2923$ (s, 
$v_{\mathrm{s}}\left(\mathrm{C}\right.$ alkyl-H), $2531\left(\mathrm{~s}, v_{\mathrm{s}}(\mathrm{B}-\mathrm{H})\right), 1480 \mathrm{~cm}-1\left(\mathrm{~s}, v_{\mathrm{s}}(\mathrm{N}-\mathrm{CH} 3)\right) .1 \mathrm{H}$ NMR $\left(\mathrm{CDCl}_{3}\right): \delta=3.11(\mathrm{~s}, 9 \mathrm{H}$,

$\left.\mathrm{NH}\left(\mathrm{CH}_{3}\right)_{3}\right), 2.18(\mathrm{~s}, 2 \mathrm{H}, \quad \mathrm{C}$ cluster- $H) . \quad{ }_{1} \mathrm{H}\{11 \mathrm{~B}\} \quad \mathrm{NMR}\left(\mathrm{CD}_{3} \mathrm{COCD}_{3}\right): \delta=3.11 \quad(\mathrm{~s}, 9 \mathrm{H}$, $\left.\mathrm{NH}\left(\mathrm{CH}_{3}\right)_{3}\right), 2.50-0.5$ (s, 8H, B-H), 2.18 (br s, 2H, Ccluster- $H$ ), -2.41 ppm (s, 1H, Hbridge). ${ }_{13} \mathrm{C}\{1 \mathrm{H}\}$ NMR $\left(\mathrm{CD}_{3} \mathrm{COCD}_{3}\right): \delta=46.7 \mathrm{ppm}\left(\mathrm{s}, \mathrm{HN}\left(\mathrm{CH}_{3}\right)_{3}\right) .11 \mathrm{~B}$ NMR $\left(\mathrm{CDCl}_{3}\right): \delta=-10.7(\mathrm{~d}$, ${ }_{1} J(\mathrm{~B}, \mathrm{H})=147,1 \mathrm{~B}, \mathrm{~B}(9),-11.7\left(\mathrm{~d},{ }_{1} J(\mathrm{~B}, \mathrm{H})=149,1 \mathrm{~B}, \mathrm{~B}(11),-17.9\left(\mathrm{~d},{ }_{1} J(\mathrm{~B}, \mathrm{H})=126,2 \mathrm{~B}\right.\right.$, $\mathrm{B}(2,4)),-20.6(\mathrm{~d}, 1 J(\mathrm{~B}, \mathrm{H})=134,1 \mathrm{~B}, \mathrm{~B}(6)),-21.8(\mathrm{~d}, 1 J(\mathrm{~B}, \mathrm{H})=140,1 \mathrm{~B}, \mathrm{~B}(3),-24.4(\mathrm{~s}, 1 \mathrm{~B}$, B(5)), -30.9 (d, $\left.{ }_{1} J(\mathrm{~B}, \mathrm{H})=84,1 \mathrm{~B}, \mathrm{~B}(10)\right),-24.4(\mathrm{~s}, 1 \mathrm{~B}, \mathrm{~B}(5)),-36.1\left(\mathrm{~d},{ }_{1} J(\mathrm{~B}, \mathrm{H})=140,1 \mathrm{~B}\right.$, B(1)).MALDI-TOFMS: $m / z=261.24$ [M, 100\%].

\section{Synthesis of [HNMe3][7-Me-5,6-I2-7,8-nido-C 2 B9H9]}

The same procedure as for the obtaining of [HNMe3][5-I-7,8-nido- $\left.\mathrm{C}_{2} \mathrm{~B}_{9} \mathrm{H}_{11}\right]$ was followed. The used reactants quantities were: $\mathrm{KOH}$ (102 mg, $1.829 \mathrm{mmol})$, EtOH (5 ml), 1-Me-9,12I2-1,2-closo- $\mathrm{C}_{2} \mathrm{~B} 10 \mathrm{H} 9(150 \mathrm{mg}, 0.365 \mathrm{mmol})$. The final white solid [HNMe3][7-Me-5,6-I27,8-nido- $\left.\mathrm{C}_{2} \mathrm{~B} 9 \mathrm{H} 9\right]$ was obtained with a yield of $87 \%(151 \mathrm{mg})$. Elemental analysis for $\mathrm{C}_{6} \mathrm{H}_{22} \mathrm{~B} 9 \mathrm{I}_{2} \mathrm{~N}$ : calc: $\mathrm{C} 15.69, \mathrm{H} 4.83, \mathrm{~N} 3.05$; found $\mathrm{C} 15.83, \mathrm{H} 4.57, \mathrm{~N} 3.13$. IR $(\mathrm{KBr}): \mathrm{v}=$ $3026(\mathrm{~N}-\mathrm{H}), 2957\left(\mathrm{C}_{\mathrm{c}-\mathrm{H}}\right), 2925$ (Calkyl-H), 1480 (N-C), 2541 (B-H).1H NMR (CD $\left.3 \mathrm{COCD}_{3}\right)$ : $\delta=3.47\left(\mathrm{~s}, 12 \mathrm{H}, \mathrm{N}\left(\mathrm{CH}_{3}\right) 4\right), 1.30\left(\mathrm{~s}, 3 \mathrm{H}, \mathrm{CH}_{3}\right) .{ }_{1} \mathrm{H}\{11 \mathrm{~B}\} \mathrm{NMR}\left(\mathrm{CD}_{3} \mathrm{COCD}_{3}\right): \delta=3.47(\mathrm{~s}$, 12H, N(CH3)4), 2.40-1.05 (s, B-H), 1.30 (s, 3H, $\left.\mathrm{CH}_{3}\right),-1.89$ (s, 1H, Hbridge). $13 \mathrm{C}\{1 \mathrm{H}\}$ NMR $\left(\mathrm{CDCl}_{3}\right): \delta=55.38\left(\mathrm{~N}\left(\mathrm{CH}_{3}\right) 4\right), 23.91\left(\mathrm{CH}_{3}\right) .11 \mathrm{~B} \mathrm{NMR}\left(\mathrm{CDCl}_{3}\right): \delta=-7.6\left(\mathrm{~d},{ }_{1} J(\mathrm{~B}, \mathrm{H})=144\right.$ 1B), $-8.8(\mathrm{~d}, 1 J(\mathrm{~B}, \mathrm{H})=140,1 \mathrm{~B}),-12.2(\mathrm{~d}, 1 J(\mathrm{~B}, \mathrm{H})=164,1 \mathrm{~B}),-16.4(\mathrm{~d}, 1 J(\mathrm{~B}, \mathrm{H})=171,1 \mathrm{~B}),-$ $20.2(\mathrm{~d}, 1 J(\mathrm{~B}, \mathrm{H})=156,1 \mathrm{~B}),-23.4($ br s, 1B) $,-24.2($ br s, 1B $),-27.3(\mathrm{~d} \mathrm{~d}, 1 J(\mathrm{~B}, \mathrm{H})=138$, $\left.{ }_{1} J(\mathrm{H}, \mathrm{H})=34,1 \mathrm{~B}\right),-31.8\left(\mathrm{~d},{ }_{1} J(\mathrm{~B}, \mathrm{H})=150,1 \mathrm{~B}\right)$.MALDI-TOFMS: $m / z=398.60$ [M, 100\%].

\section{Synthesis of [HNMe3][7-Ph-5,6-I2-nido-7,8-C2B9H9]}


The same procedure as for the obtaining of [HNMe3][5-I-7,8-nido- $\left.\mathrm{C}_{2} \mathrm{~B} 9 \mathrm{H}_{11}\right]$ was followed. The used reactants quantities were: $\mathrm{KOH}(0.5 \mathrm{~g}, 1.06 \mathrm{mmol})$, EtOH (15 ml), 1-Ph-9,12-I21,2-closo- $\mathrm{C}_{2} \mathrm{~B}_{10} \mathrm{H}_{9}(500 \mathrm{mg}, 1.06 \mathrm{mmol})$. The final white solid product, [HNMe3][7-Ph-5,6$\mathrm{I}_{2}-7,8-$ nido- $\left.\mathrm{C}_{2} \mathrm{~B} 9 \mathrm{H} 9\right]$ was obtained with a yield of $73 \%(395 \mathrm{mg})$. Elemental analysis for $\mathrm{C}_{11} \mathrm{H}_{24} \mathrm{~B} 9 \mathrm{I}_{2} \mathrm{~N}$ : calc: $\mathrm{C} 25.34, \mathrm{H} 4.64, \mathrm{~N} 2.69$; found $\mathrm{C} 25.23, \mathrm{H} 4.81, \mathrm{~N} 2.42$. IR (KBr): $v=$ $3077(\mathrm{~N}-\mathrm{H}), 3026\left(\mathrm{C}_{\mathrm{c}-\mathrm{H}}\right), 2543(\mathrm{~B}-\mathrm{H}) 1479(\mathrm{~N}-\mathrm{C}) .1 \mathrm{H}$ NMR (CD $\left.{ }_{3} \mathrm{COCD}_{3}\right): \delta=7.22-7.07$ $(\mathrm{m}, 5 \mathrm{H}, \mathrm{C} 6 \mathrm{H} 5), \delta=3.46\left(\mathrm{~s}, 12 \mathrm{H}, \mathrm{N}\left(\mathrm{CH}_{3}\right)_{4}\right) .1 \mathrm{H}\{11 \mathrm{~B}\} \mathrm{NMR}\left(\mathrm{CD}_{3} \mathrm{COCD}_{3}\right): \delta=7.22-7.07(\mathrm{~m}$, $5 \mathrm{H}, \mathrm{C} 6 \mathrm{H} 5), \delta=3.46\left(\mathrm{~s}, 12 \mathrm{H}, \mathrm{N}\left(\mathrm{CH}_{3}\right) 4\right), 2.81-1.24(\mathrm{~s}, \mathrm{~B}-\mathrm{H}),-1.58(\mathrm{~s}, 1 \mathrm{H}, \mathrm{Hbridge}) .{ }_{13} \mathrm{C}\{1 \mathrm{H}\}$ NMR $\left(\mathrm{CDCl}_{3}\right): \delta=55.35\left(\mathrm{~N}\left(\mathrm{CH}_{3}\right) 4\right), 127.56,126.65,125.22\left(\mathrm{C}_{6} \mathrm{H} 5\right) .11 \mathrm{~B} \mathrm{NMR}\left(\mathrm{CDCl}_{3}\right): \delta=$ $-6.4\left(\mathrm{~d},{ }_{1} J(\mathrm{~B}, \mathrm{H})=153,1 \mathrm{~B}\right),-8.1\left(\mathrm{~d},{ }_{1} J(\mathrm{~B}, \mathrm{H})=151,1 \mathrm{~B}\right),-12.3\left(\mathrm{~d},{ }_{1} J(\mathrm{~B}, \mathrm{H})=162,1 \mathrm{~B}\right),-17.7$ $\left(\mathrm{d},{ }_{1} J(\mathrm{~B}, \mathrm{H})=162,1 \mathrm{~B}\right),-20.4\left(\mathrm{~d},{ }_{1} J(\mathrm{~B}, \mathrm{H})=152,1 \mathrm{~B}\right),-22.5(\mathrm{br} \mathrm{s}, 1 \mathrm{~B}),-23.8(\mathrm{br} \mathrm{s}, 1 \mathrm{~B}),-26.4$

$(\mathrm{d} \mathrm{d}, 1 J(\mathrm{~B}, \mathrm{H})=150),-30.9\left(\mathrm{~d},{ }_{1} J(\mathrm{~B}, \mathrm{H})=147,1 \mathrm{~B}\right)$. MALDI-TOF MS: $m / z=462.81[\mathrm{M}$, $100 \%]$.

\section{Synthesis of [HNMe3][7-Me-1,5,6,10-I4-7,8-nido- $\left.\mathrm{C}_{2} \mathrm{~B}_{9} \mathrm{H}_{7}\right]$}

The same procedure as for the obtaining of [HNMe3][5-I-7,8-nido- $\left.\mathrm{C}_{2} \mathrm{~B}_{9} \mathrm{H}_{11}\right]$ was followed.

The used reactants quantities were: $\mathrm{KOH}(0.63 \mathrm{~g}, 1.13 \mathrm{mmol})$, EtOH $(5 \mathrm{ml}), 1-\mathrm{Me}-$ 8,9,10,12-I4-1,2-closo- $\mathrm{C}_{2} \mathrm{~B}_{10} \mathrm{H}_{7}(150 \mathrm{mg}, 0.226 \mathrm{mmol})$. The final white solid product, [HNMe3][7-Me-1,5,6,10-I4-7,8-nido- $\mathrm{C}_{2} \mathrm{~B} 9 \mathrm{H} 7$ ], was obtained with a yield of 63\% (100 mg). Elemental analysis for $\mathrm{C}_{6} \mathrm{H}_{20} \mathrm{~B} 9 I_{4} \mathrm{~N}$ : calc: $\mathrm{C} 10.13, \mathrm{H} 2.83, \mathrm{~N}$ 1.97; found C 10.07, H 2.65, N 1.80. IR (KBr): v= $3115(\mathrm{~s}, v(\mathrm{~N}-\mathrm{H})), 2923(\mathrm{w}, \mathrm{v}($ Ccluster-H st. $)), 2865$ (w, $v($ Calkyl-H st. $))$, 2555 (s, v(B-H st.)), $1462(\mathrm{w}, v(\mathrm{~N}-\mathrm{C})) .1 \mathrm{H}-\mathrm{NMR}:\left(\mathrm{CD}_{3} \mathrm{COCD}_{3}\right) \delta=8.51,\left(\mathrm{t},{ }_{1} \mathrm{~J}(\mathrm{~N}, \mathrm{H})=111\right.$, $\left.1 \mathrm{H}, \mathrm{NH}\left(\mathrm{CH}_{3}\right)_{3}\right), 3.2\left(\mathrm{~d}, 3 \mathrm{~J}(\mathrm{H}, \mathrm{H})=3,9 \mathrm{H}, \mathrm{NH}\left(\mathrm{CH}_{3}\right)_{3}\right), 2.37(\mathrm{~s}, 3 \mathrm{H}, \mathrm{CH} 3$ cluster). $1 \mathrm{H}\{11 \mathrm{~B}\} \mathrm{NMR}$ $\left(\mathrm{CD}_{3} \mathrm{COCD}_{3}\right): \delta=8.51\left(\mathrm{t}, 1 \mathrm{~J}(\mathrm{~N}, \mathrm{H})=111,1 \mathrm{H}, \mathrm{NH}\left(\mathrm{CH}_{3}\right)_{3}\right), 3.2(\mathrm{~d}, 3 \mathrm{~J}(\mathrm{H}, \mathrm{H})=3,9 \mathrm{H}$, 
$\left.\mathrm{NH}\left(\mathrm{CH}_{3}\right)_{3}\right), 2.60$ (m, 4H, B-H), 2.37 (s, 3H, CH3 cluster), 2.19 (s, 1H, B-H), 0.19 (s, 1H, Hbridge). $13 \mathrm{C}\{1 \mathrm{H}\} \quad \mathrm{NMR} \quad\left(\mathrm{CD}_{3} \mathrm{COCD}_{3}\right): \delta=45.5 \quad\left(\mathrm{~s}, \mathrm{HN}\left(\mathrm{CH}_{3}\right)_{3}\right), 23.5 \quad\left(\mathrm{CH}_{3}\right)_{111 \mathrm{~B}} \mathrm{NMR}$ $\left(\mathrm{CD}_{3} \mathrm{COCD}_{3}\right): \delta=-7.50\left(\mathrm{~d},{ }_{1} \mathrm{~J}(\mathrm{~B}, \mathrm{H})=144,1 \mathrm{~B}\right),-8.73\left(\mathrm{~d},{ }_{1} \mathrm{~J}(\mathrm{~B}, \mathrm{H})=131,1 \mathrm{~B}\right),-12.18\left(\mathrm{~d},{ }_{1} \mathrm{~J}\right.$ $(B, H)=195,1 B),-14.21(\mathrm{~d}, 1 \mathrm{~J}(\mathrm{~B}, \mathrm{H})=160,1 \mathrm{~B}),-17.97(\mathrm{~s}, 2 \mathrm{~B}, \mathrm{~B}-\mathrm{I}),-17.97(\mathrm{~d}, 1 \mathrm{~J}(\mathrm{~B}, \mathrm{H})=$ 93, 1B), -35.23 (s, 2B, B-I). MALDI-TOF MS: m/z= 524.98 [M-I, 24\%], $651.40[\mathrm{M}$, $100 \%]$.

\section{Synthesis of [HNMe3][7-Ph-1,5,6,10-I4-7,8-nido- $\mathrm{C}_{2} \mathrm{~B} 9 \mathrm{H}$ 7]}

The same procedure as for the obtaining of [HNMe3][5-I-7,8-nido- $\left.\mathrm{C}_{2} \mathrm{~B}_{9} \mathrm{H}_{11}\right]$ was followed.

The used reactants quantities were: $\mathrm{KOH}(0.77 \mathrm{~g}, 1.38 \mathrm{mmol})$, EtOH $(5 \mathrm{ml}), 1-\mathrm{Ph}-$ 8,9,10,12-I4-1,2-closo- $\mathrm{C}_{2} \mathrm{~B}_{10} \mathrm{H}_{7}$ (200 $\left.\mathrm{mg}, 0.27 \mathrm{mmol}\right)$. The final white solid product, [HNMe3][7-Ph-1,5,6,10-I4-7,8-nido- $\left.\mathrm{C}_{2} \mathrm{~B} 9 \mathrm{H} 7\right]$, was obtained with a yield of $73 \%$ (156 mg). Elemental analysis for $\mathrm{C}_{11} \mathrm{H}_{22} \mathrm{~B} 9 \mathrm{I}_{4} \mathrm{~N}$ : calc: C 17.09, H 2.87, N 1.81; found C 17.20, H 2.97, N 1.74. IR (KBr): $v=3111(\mathrm{~s}, v(\mathrm{~N}-\mathrm{H})), 2548(\mathrm{~s}, v(\mathrm{~B}-\mathrm{H}$ st.)), $1463(\mathrm{w}, v(\mathrm{~N}-\mathrm{C}))$. $1 \mathrm{HNMR}$ $\left(\mathrm{CD}_{3} \mathrm{COCD}_{3}\right): \delta=8.71\left(\mathrm{~s}, 1 \mathrm{~J}(\mathrm{~N}, \mathrm{H})=102,1 \mathrm{H}, \mathrm{NH}\left(\mathrm{CH}_{3}\right) 3\right), 7.23-7.17(\mathrm{~m}, 5 \mathrm{H}, \mathrm{C} 6 \mathrm{H} 5), 3.19(\mathrm{~d}$, $\left.{ }_{3} \mathrm{~J}(\mathrm{H}, \mathrm{H})=6,9 \mathrm{H}, \mathrm{NH}\left(\mathrm{CH}_{3}\right)_{3}\right) .1 \mathrm{H}\{11 \mathrm{~B}\} \operatorname{NMR}\left(\mathrm{CD}_{3} \mathrm{COCD}_{3}\right): \delta=8.71\left(\mathrm{~s},{ }_{1} \mathrm{~J}(\mathrm{~N}, \mathrm{H})=102,1 \mathrm{H}\right.$, $\left.\mathrm{NH}\left(\mathrm{CH}_{3}\right) 3\right), 7.23-7.17\left(\mathrm{~m}, 5 \mathrm{H}, \mathrm{C}_{6} \mathrm{H}_{5}\right), 3.19\left(\mathrm{~d},{ }_{3} \mathrm{~J}(\mathrm{H}, \mathrm{H})=6,9 \mathrm{H}, \mathrm{NH}\left(\mathrm{CH}_{3}\right) 3\right), 2.93-1.2(\mathrm{~m}$, 5H, B-H), 0.4 (s, 1H, Hbridge). ${ }_{13} \mathrm{C}\{1 \mathrm{H}\}$ NMR ( $\left.\mathrm{CD}_{3} \mathrm{COCD}_{3}\right): \delta=141.7$ (Cipso of $\left.\mathrm{C}_{6} \mathrm{H}_{5}\right), 128.0$, 126.6, $126.1\left(\mathrm{C}_{6} \mathrm{H} 5\right), 45.5\left(\mathrm{~s}, \mathrm{HN}\left(\mathrm{CH}_{3}\right)_{3}\right) .11 \mathrm{~B}$ NMR $\left(\mathrm{CD}_{3} \mathrm{COCD}_{3}\right): \delta=-6.5\left(\mathrm{~d},{ }_{1} \mathrm{~J}(\mathrm{~B}, \mathrm{H})=\right.$ 147, 1B), -8.0 (d, $\left.{ }_{1} \mathrm{~J}(\mathrm{~B}, \mathrm{H})=148,1 \mathrm{~B}\right),-12.4(\mathrm{~s}, 1 \mathrm{~B}, \mathrm{~B}-\mathrm{I}),-15.5(\mathrm{~d}, 1 \mathrm{~J}(\mathrm{~B}, \mathrm{H})=135,1 \mathrm{~B}),-$ $17.2(\mathrm{~m}, 1 \mathrm{~B}, \mathrm{~B}-\mathrm{I}),-18.2(\mathrm{~m}, 2 \mathrm{~B}, \mathrm{~B}-\mathrm{H}),-34.5$ (s, 2B, B-I, B(1,10)). MALDI-TOF-MS: m/z= 587 [M-I, 55\%], 713 [M, 100\%].

\section{Synthesis of [HNMe3][5,6,9-I3-7,8-nido-C2B9H9]}


In a $100 \mathrm{~mL}$ round-bottom flask was placed a magnetic stir bar, [HNMe3][5,6-I2-7,8-nido$\mathrm{C}_{2} \mathrm{~B}_{9} \mathrm{H}_{10}$ ] (508 $\left.\mathrm{mg}, 1,14 \mathrm{mmol}\right)$ and $20 \mathrm{~mL}$ of $\mathrm{MeOH}$. A solution of $\mathrm{I}_{2}(579 \mathrm{mg}, 2,28 \mathrm{mmol}$, 2 eq) in $20 \mathrm{~mL}$ of $\mathrm{MeOH}$ was added to the reaction flask and the mixture was refluxed overnight. Addition of $10 \mathrm{~mL}$ of $\mathrm{H}_{2} \mathrm{O}$ and subsequent evaporation of the $\mathrm{MeOH} / \mathrm{H}_{2} \mathrm{O}$ solvent resulted in the precipitation of a white solid from the aqueous layer. Recrystallization from boiling $\mathrm{H}_{2} \mathrm{O}$ gave the desired compound [HNMe3][5,6,9-I3-7,8-nido$\left.\mathrm{C}_{2} \mathrm{~B} 9 \mathrm{H} 9\right](84 \%, 548 \mathrm{mg})$. Elemental analysis for $\mathrm{C}_{5} \mathrm{H}_{19}{ }_{19}{ }_{9}{ }_{3} \mathrm{~N}$ : calc: $\mathrm{C} 10.51, \mathrm{H} 3.35, \mathrm{~N} 2.45$; found C 10.50, H 3.37, N 2.21. IR (KBr): v= $3133(\mathrm{~s}, v(\mathrm{~N}-\mathrm{H})), 2591,2565,2539(\mathrm{~s}, \mathrm{v}(\mathrm{B}-\mathrm{H}$ st.)), $1461(\mathrm{w}, v(\mathrm{~N}-\mathrm{C})), 970.1 \mathrm{H}$ NMR $\left(\mathrm{CD}_{3} \mathrm{COCD}_{3}\right): \delta=8.51\left(\mathrm{br} \mathrm{t},{ }_{1} \mathrm{~J}(\mathrm{~N}, \mathrm{H})=4.9,1 \mathrm{H}, \mathrm{N}-\mathrm{H}\right)$, $3.23\left(\mathrm{~s}, 9 \mathrm{H}, \mathrm{NH}\left(\mathrm{CH}_{3}\right)_{3}\right), 2.68($ br s, $1 \mathrm{H}, \mathrm{Cc}-\mathrm{H}), 2.30$ (br s, 1H, Cc-H). $1 \mathrm{H}\{11 \mathrm{~B}\}$ NMR $\left(\mathrm{CD}_{3} \mathrm{COCD}_{3}\right): \delta=8.51\left(\mathrm{br} \mathrm{t},{ }_{1} \mathrm{~J}(\mathrm{~N}, \mathrm{H})=4.9,1 \mathrm{H}, \mathrm{N}-\mathrm{H}\right), 3.23\left(\mathrm{~s}, 9 \mathrm{H}, \mathrm{NH}\left(\mathrm{CH}_{3}\right)_{3}\right), 2.68(\mathrm{br} \mathrm{s}$, 1H, Cc-H), 2.55 (br s, B-H), 2.30 (br s, 1H, Cc-H), 1.87 (br s, B-H), $1.34(\mathrm{~d}, 1 \mathrm{~J}(\mathrm{H}, \mathrm{H})=9$, 1H, B(10)-H), $1.24(\mathrm{~s}, 1 \mathrm{H}, \mathrm{B}(1)-\mathrm{H}),-2.25(\mathrm{t}, 3 \mathrm{~J}(\mathrm{H}, \mathrm{H})=9,1 \mathrm{H}, \mathrm{BHB}) .{ }_{13} \mathrm{C}\{1 \mathrm{H}\} \quad \mathrm{NMR}$ ( $\mathrm{CD}_{3} \mathrm{COCD}_{3}$ ): 51.28-49.61 (br m, $\mathrm{Cc}$ ), 45.51 (s, $\left.\mathrm{HN}\left(\mathrm{CH}_{3}\right)_{3}\right), 37,31-35.87$ (br m, Ccluster). ${ }_{11} \mathrm{~B}$ $\operatorname{NMR}\left(\mathrm{CD}_{3} \mathrm{COCD}_{3}\right):-12.6($ br s, 2B), -15.7 (d, $1 \mathrm{~J}(\mathrm{~B}, \mathrm{H})=167,2 \mathrm{~B}),-15.7(\mathrm{~s}, 1 \mathrm{~B}),-19.1(\mathrm{~d}$, $\left.{ }_{1} \mathrm{~J}(\mathrm{~B}, \mathrm{H})=162,1 \mathrm{~B}\right),-23.2\left(\mathrm{~d},{ }_{1} \mathrm{~J}(\mathrm{~B}, \mathrm{H})=136,1 \mathrm{~B}\right),-30.3(\mathrm{~s}, 1 \mathrm{~B}), 31.7\left(\mathrm{~d},{ }_{1} \mathrm{~J}(\mathrm{~B}, \mathrm{H})=146\right.$, 1B). MALDI-TOF-MS: $\mathrm{m} / \mathrm{z}=510.87$ [M, 100\%].

\section{Synthesis of [NMe4][5,6-Me2-7,8-nido- $\left.\mathrm{C}_{2} \mathrm{~B}_{9} \mathrm{H}_{10}\right]$}

To a solution of $\mathrm{KOH}(162 \mathrm{mg}, 2.9 \mathrm{mmol})$ in degassed EtOH (4 mL) 9,12-Me2-1,2-closo$\mathrm{C}_{2} \mathrm{~B}_{10} \mathrm{H}_{10}(100 \mathrm{mg}, 0.58 \mathrm{mmol})$ was added. The solution was refluxed for $20 \mathrm{~h}$. After cooling down to room temperature, the solvent was removed under reduced pressure and the solid residue was dissolved in $5 \mathrm{~mL}$ of water. The solution was neutralized with $\mathrm{HCl}$ 1M. Afterwards, aqueous solution of $\left[\mathrm{NMe}_{4}\right] \mathrm{Cl}$ was added dropwise until no more 
precipitate was formed. The white solid was rinsed with water and diethyl ether to give [NMe4][5,6-Me2-7,8-nido-C2 $\left.{ }_{2}{ }_{9} \mathrm{H}_{10}\right](77 \%, 106 \mathrm{mg}) . \mathrm{IR}(\mathrm{KBr}): \mathrm{v}=3048,3040\left(\mathrm{~s}, v_{\mathrm{s}}(\mathrm{C} \mathrm{c}-\mathrm{H})\right)$, 2925, 2895, 2832 (w, v(Calkyl-H st.)), 2501, 2490 (s, v(B-H st.)), 1482(vs(N-CH3)), 945 (s, Vas $\left.\left(\mathrm{CH}_{3}\right)\right)$. ${ }_{1} \mathrm{HNMR}\left(\mathrm{CD}_{3} \mathrm{COCD}_{3}\right): 3.46$ (s, 12H, N(CH3)4), 1.53 (br s, 2H; Ccluster-H), -0.04 $\left(\mathrm{s}, 6 \mathrm{H}, \mathrm{CH}_{3}\right) ;{ }_{1} \mathrm{H}\{11 \mathrm{~B}\} \mathrm{NMR}\left(\mathrm{CD}_{3} \mathrm{COCD}_{3}\right): \delta=3.46\left(\mathrm{~s}, 12 \mathrm{H}, \mathrm{N}\left(\mathrm{CH}_{3}\right) 4\right), 1.53$ (br s, $2 \mathrm{H}$; Ccluster-H), 1.96, 1.29, 0.37, 0.19 (br s, B-H), -0.04 (s, 6H, CH3); -2.47 (d, ${ }_{1} \mathrm{~J}(\mathrm{H}, \mathrm{H})=9,1 \mathrm{H}$, Hbridge). ${ }_{13} \mathrm{C}\left\{{ }_{1} \mathrm{H}\right\}$ NMR $\left(\mathrm{CD}_{3} \mathrm{COCD}_{3}\right): 37.62\left(\mathrm{qu},{ }_{1} \mathrm{~J}(\mathrm{C}, \mathrm{B})=25, \mathrm{Cc}_{\mathrm{c}}\right), 55.32(\mathrm{~s}, \mathrm{~N}(\mathrm{CH} 3) 4)$, 2.31-0.00 (br m, B-CH3). 11B NMR ( $\left.\mathrm{CD}_{3} \mathrm{COCD}_{3}\right)$ : $\delta=-6.2\left(\mathrm{~s}, 2 \mathrm{~B}, \mathrm{~B}-\mathrm{CH}_{3}, \mathrm{~B}(5,6)\right),-8.5(\mathrm{~d}$, $\left.{ }_{1} \mathrm{~J}(\mathrm{~B}, \mathrm{H})=131,2 \mathrm{~B}, \mathrm{~B}(9,11)\right),-17.2\left(\mathrm{~d},{ }_{1} \mathrm{~J}(\mathrm{~B}, \mathrm{H})=163,1 \mathrm{~B}, \mathrm{~B}(3)\right),-19.7\left(\mathrm{~d},{ }_{1} \mathrm{~J}(\mathrm{~B}, \mathrm{H})=145\right.$ $2 \mathrm{~B}, \mathrm{~B}(2,4)),-27.5(\mathrm{~d}, 1 \mathrm{~J}(\mathrm{~B}, \mathrm{H})=130,1 \mathrm{~B}, \mathrm{~B}(10)),-32.8\left(\mathrm{~d},{ }_{1} \mathrm{~J}(\mathrm{~B}, \mathrm{H})=134,1 \mathrm{~B}, \mathrm{~B}(1)\right)$. MALDI-TOF-MS: m/z=161.23 [M, 100\%].

\section{Synthesis of [NBu4][1,5,6,10-Me4-7,8-nido-C2B9H8]}

A stirred mixture of 8,9,10,12-Me4-1,2-closo- $\mathrm{C}_{2} \mathrm{~B}_{10} \mathrm{H}_{8}(150 \mathrm{mg}, 0.74 \mathrm{mmol})$ and $\mathrm{Bu} 4 \mathrm{NF}$ hydrate $(978 \mathrm{mg}, 3.74 \mathrm{mmol})$ in THF $(10 \mathrm{~mL})$ was heated under reflux for 4 hours. After cooling, the solvent was removed. The solid was dissolved in dichloromethane, washed with water $(100 \mathrm{~mL})$, dried over $\mathrm{MgSO}_{4}$ and the solvent vaccum removed. The resulting oil was washed with hexane to give $[\mathrm{NBu} 4]\left[1,5,6,10-\mathrm{Me} 4-7,8-\right.$ nido- $\left.\mathrm{C}_{2} \mathrm{~B} 9 \mathrm{H} 8\right]$ as a white solid (298 mg, 92\%). IR (KBr): v=2964, 2933, 2895, 2877, 2831 (s, v(Calkyl-H)), 2527, 2496 (s, $v(\mathrm{~B}-\mathrm{H}$ st.) $), 1471,974(\mathrm{w}, \mathrm{v}(\mathrm{N}-\mathrm{C})) .{ }_{1} \mathrm{H}$ NMR $\left(\mathrm{CD}_{3} \mathrm{COCD}_{3}\right): \delta=3.45(\mathrm{t}, 3 \mathrm{~J}(\mathrm{H}, \mathrm{H})=9,8 \mathrm{H}$, $\left.\left(\mathrm{CH}_{3} \mathrm{CH}_{2} \mathrm{CH}_{2} \mathrm{CH}_{2}\right)_{4} \mathrm{~N}\right), \quad 1.83 \quad\left(\mathrm{~m}, \quad 3 \mathrm{~J}(\mathrm{H}, \mathrm{H})=9, \quad 8 \mathrm{H}, \quad\left(\mathrm{CH}_{3} \mathrm{CH}_{2} \mathrm{CH}_{2} \mathrm{CH}_{2}\right) 4 \mathrm{~N}\right), \quad 1.46 \quad(\mathrm{~m}$, $\left.{ }_{3} \mathrm{~J}(\mathrm{H}, \mathrm{H})=6,8 \mathrm{H}, \quad\left(\mathrm{CH}_{3} \mathrm{CH}_{2} \mathrm{CH}_{2} \mathrm{CH}_{2}\right) 4 \mathrm{~N}\right), 0.99\left(\mathrm{t},{ }_{3} \mathrm{~J}(\mathrm{H}, \mathrm{H})=6,12 \mathrm{H},\left(\mathrm{CH}_{3} \mathrm{CH}_{2} \mathrm{CH}_{2} \mathrm{CH}_{2}\right)_{4} \mathrm{~N}\right)$, 0.05 (br s, 3H, $\left.\mathrm{CH}_{3}\right),-0.29$ (br s, 9H, CH 3$) .1 \mathrm{H}\{11 \mathrm{~B}\}$ NMR $\left(\mathrm{CD}_{3} \mathrm{COCD}_{3}\right): \delta=3.45(\mathrm{t}$ $\left.3 \mathrm{~J}(\mathrm{H}, \mathrm{H})=9, \quad 8 \mathrm{H}, \quad\left(\mathrm{CH}_{3} \mathrm{CH}_{2} \mathrm{CH}_{2} \mathrm{CH}_{2}\right)_{4} \mathrm{~N}\right), \quad 1.84 \quad\left(\mathrm{~m}, \quad 3 \mathrm{~J}(\mathrm{H}, \mathrm{H})=9, \quad{ }_{3} \mathrm{~J}(\mathrm{H}, \mathrm{H})=6, \quad 8 \mathrm{H}\right.$, 
$\left.\left(\mathrm{CH}_{3} \mathrm{CH}_{2} \mathrm{CH}_{2} \mathrm{CH}_{2}\right)_{4} \mathrm{~N}\right), 1.73($ br s, 2H, B-H $), 1.46(\mathrm{~m}, 3 \mathrm{~J}(\mathrm{H}, \mathrm{H})=9,3 \mathrm{~J}(\mathrm{H}, \mathrm{H})=6,8 \mathrm{H}$, $\left.\left(\mathrm{CH}_{3} \mathrm{CH}_{2} \mathrm{CH}_{2} \mathrm{CH}_{2}\right)_{4} \mathrm{~N}\right), 1.13 \quad($ br $\quad \mathrm{s}, \quad 3 \mathrm{H}, \quad \mathrm{B}-\mathrm{H}), \quad 0.99 \quad\left(\mathrm{t}, \quad{ }_{3} \mathrm{~J}(\mathrm{H}, \mathrm{H}) \quad=6, \quad 12 \mathrm{H}\right.$, $\left.\left(\mathrm{CH}_{3} \mathrm{CH}_{2} \mathrm{CH}_{2} \mathrm{CH}_{2}\right) 4 \mathrm{~N}\right), 0.05\left(\mathrm{~s}, 3 \mathrm{H}, \mathrm{CH}_{3}\right),-0.28\left(\mathrm{~s}, 6 \mathrm{H}, \mathrm{CH}_{3}\right),-0.31\left(\mathrm{~s}, 3 \mathrm{H}, \mathrm{CH}_{3}\right),-1.87$ (s, 1H, Hbridge). ${ }_{13} \mathrm{C}\{1 \mathrm{H}\}$ NMR $\left.\left(\mathrm{CD}_{3} \mathrm{COCD}_{3}\right): 58.5\left(\mathrm{~s}, \mathrm{CH}_{3} \mathrm{CH}_{2} \mathrm{CH}_{2} \mathrm{CH}_{2}\right) 4 \mathrm{~N}+\right), 37.81\left(\mathrm{~m}, \mathbf{C}_{\mathrm{cH}}\right)$, 23.5 (s, $\left.\left.\left.\quad \mathrm{CH}_{3} \mathrm{CH}_{2} \mathrm{CH}_{2} \mathrm{CH}_{2}\right) 4 \mathrm{~N}_{+}\right), \quad 19.4 \quad\left(\mathrm{~s}, \quad \mathrm{CH}_{3} \mathrm{CH}_{2} \mathrm{CH}_{2} \mathrm{CH}_{2}\right)_{4} \mathrm{~N}+\right), \quad 12.9 \quad$ (s, $\left.\left.\mathrm{CH}_{3} \mathrm{CH}_{2} \mathrm{CH}_{2} \mathrm{CH}_{2}\right){ }_{4} \mathrm{~N}+\right),-0.6 /-1.9$ (br s, $\left.\mathrm{BCH}_{3}\right)$. ${ }_{11} \mathrm{~B}$ NMR $\left(\mathrm{CD}_{3} \mathrm{COCD}_{3}\right): \delta=-6.6(\mathrm{~s}, 2 \mathrm{~B}, \mathrm{~B}-$ $\left.\mathrm{CH}_{3}\right),-8.7\left(\mathrm{~d},{ }_{1} \mathrm{~J}(\mathrm{~B}, \mathrm{H})=132,2 \mathrm{~B}\right),-18.0\left(\mathrm{~d},{ }_{1} \mathrm{~J}(\mathrm{~B}, \mathrm{H})=173,1 \mathrm{~B}\right),-19.9\left(\mathrm{~d},{ }_{1} \mathrm{~J}(\mathrm{~B}, \mathrm{H})=155\right.$, 2B), $-22.6\left(\mathrm{~s}, 1 \mathrm{~B}, \mathrm{~B}-\mathrm{CH}_{3}\right),-27.0$ (s, 1B, B-CH3). MALDI-TOF-MS: $\mathrm{m} / \mathrm{z}=190,29$ [M, $100 \%]$.

\section{Synthesis of [NHMe3][3,3'-Co-(9-I-1,2-closo-C2 $\left.\left.\mathrm{B}_{9} \mathrm{H}_{10}\right)_{2}\right]$}

The same procedure as for the obtaining of compound [NMe4][3,3'-Co-(1-Me-9,12-I2-1,2closo- $\left.\left.\mathrm{C}_{2} \mathrm{~B} 9 \mathrm{H} 8\right)_{2}\right]$ was followed. The used reactants quantities were: [HNMe3][5-I-7,8-nido$\left.\mathrm{C}_{2} \mathrm{~B}_{9} \mathrm{H}_{11}\right]$ ( $\left.8.2 \mathrm{mg}, 0.45 \mathrm{mmol}\right)$, THF $(10 \mathrm{~mL})$, solution of butyllithium in hexanes $(0.98 \mathrm{~mL}$, $1.4 \mathrm{M}, 1.37 \mathrm{mmol}), \mathrm{CoCl}_{2}(356.8 \mathrm{mg}, 2.74 \mathrm{mmol}), 10 \mathrm{~mL}$ of diethyl ether, $10 \mathrm{~mL}$ of $\mathrm{HCl}$ $(37 \%, 0.25 \mathrm{M})$ aqueous solution. The final orange solid [NHMe3][3,3'-Co-(9-I-1,2-closo$\left.\left.\mathrm{C}_{2} \mathrm{~B}_{9} \mathrm{H}_{10}\right)_{2}\right]$ was obtained with a yield of $74 \%(130 \mathrm{mg})$. Elemental analysis for $\mathrm{C}_{7} \mathrm{H}_{30} \mathrm{~B}_{18} \mathrm{CoI}_{2} \mathrm{~N}$ : calc: C 13.23, H 4.76, N 2.20; found C 13.18, H 4.97, N 2.31. IR (ATR): $v=3022\left(\mathrm{~s}, v_{\mathrm{s}}\left(\mathrm{C}_{\text {cluster-H}} \mathrm{H}\right)\right), 2955,2924,2855\left(\mathrm{~s}, v_{\mathrm{s}}(\mathrm{C}\right.$ alkyl-H$), 2545\left(\mathrm{vs}, v_{\mathrm{s}}(\mathrm{B}-\mathrm{H})\right), 1473\left(v_{\mathrm{s}}(\mathbf{N}-\right.$ CH3)). $1 \mathrm{H}$ NMR ( $\left.\mathrm{CD}_{3} \mathrm{COCD}_{3}\right): \delta=4.26$ (br s, 2H, Ccluster- $H$ ), 4.16 (br s, 2H, Ccluster- $H$ ), 3.09 (s, 9H, $\left.\mathrm{NH}\left(\mathrm{CH}_{3}\right)_{3}\right) .1{ }_{1} \mathrm{H}\{11 \mathrm{~B}\} \mathrm{NMR}\left(\mathrm{CD}_{3} \mathrm{COCD}_{3}\right): \delta=4.26$ (br s, $2 \mathrm{H}, \mathrm{C}$ cluster- $\left.H\right), 4.16$ (br s, $2 \mathrm{H}, \mathrm{C}$ cluster- $H), 3.09$ (s, 9H, $\left.\mathrm{NH}\left(\mathrm{CH}_{3}\right) 3\right), 3.95-1.62(\mathrm{~s}, 16 \mathrm{H}, \mathrm{B}-H) .{ }_{13} \mathrm{C}\{1 \mathrm{H}\} \quad \mathrm{NMR}$ $\left.\left(\mathrm{CD}_{3} \mathrm{COCD}_{3}\right): \delta=67.15\left(C_{\text {cluster }}\right), 44.8\left(\mathrm{~s}, \mathrm{HN}\left(\mathrm{CH}_{3}\right) 3\right)\right) .{ }_{11} \mathrm{~B} \mathrm{NMR}\left(\mathrm{CD}_{3} \mathrm{COCD}_{3}\right): \delta=7.3(\mathrm{~d}$, 
$\left.{ }_{1} J(\mathrm{~B}, \mathrm{H})=147,2 \mathrm{~B}\right), 4.0\left(\mathrm{~d},{ }_{1} J(\mathrm{~B}, \mathrm{H})=143,2 \mathrm{~B}\right),-4.4\left(\mathrm{~d}, 8 \mathrm{~B},{ }_{1} J(\mathrm{~B}, \mathrm{H})=105\right),-16.9(\mathrm{~s}, 4 \mathrm{~B}),-21.9$

$(\mathrm{d}, 2 \mathrm{~B}, 1 J(\mathrm{~B}, \mathrm{H})=166)$. MALDI-TOF MS: $m / z=575.8[\mathrm{M}, 100 \%]$.

\section{Synthesis of [NMe4][3,3'-Co-(4-I-1,2-closo-C2 2 $\left.\left.9 \mathrm{H}_{10}\right)_{2}\right]$}

The same procedure as for the obtaining of compound [NMe4][3,3'-Co-(1-Me-9,12-I2-1,2closo- $\left.\left.\mathrm{C}_{2} \mathrm{~B} 9 \mathrm{H}_{8}\right)_{2}\right]$ was followed. The crude of reaction was refluxed for 36 hours. The used reactants quantities were: [HNMe3][9-I-7,8-nido- $\left.\mathrm{C}_{2} \mathrm{~B}_{9} \mathrm{H}_{11}\right]$ (400 mg, $\left.1.26 \mathrm{mmol}\right)$, THF (20 $\mathrm{ml}$ ), solution of $\mathrm{K} t \mathrm{BuO}$ in THF (7.6 ml, 1M, 7.6mmol), $\mathrm{CoCl}_{2}(1313 \mathrm{mg}, 10.11 \mathrm{mmol}), 10 \mathrm{ml}$ of THF, $10 \mathrm{ml}$ of $\mathrm{HCl}(37 \%, 0.25 \mathrm{M})$ aqueous solution. The final orange solid compound, [NMe4][3,3'-Co-(4-I-1,2-closo- $\left.\left.\mathrm{C}_{2} \mathrm{~B}_{9} \mathrm{H}_{10}\right)_{2}\right]$ was obtained with a yield of $62 \%(260 \mathrm{mg})$, after purification on silica gel column chromatography, using $\mathrm{CH}_{2} \mathrm{Cl}_{2}$ for elution. Elemental analysis for $\mathrm{C}_{8} \mathrm{H}_{32} \mathrm{~B} 18 \mathrm{CoI}_{2} \mathrm{~N}$ : calc: C 14.79, H 4.96, N 2.16; found C 14.54, H 4.97, N 2.31. IR (ATR): v= $3029\left(\mathrm{~s}, v_{\mathrm{s}}\left(\mathrm{C}_{\text {cluster-H}}\right)\right), 2959,2929\left(\mathrm{~s}, v_{\mathrm{s}}(\mathrm{C}\right.$ alkyl-H), 2537 (vs, vs(B-H)), 1473 $\left(v_{s}(\mathrm{~N}-\mathrm{CH} 3)\right)$. ${ }_{1} \mathrm{H} \mathrm{NMR}\left(\mathrm{CD}_{3} \mathrm{COCD}_{3}\right): \delta=4.69\left(\mathrm{~s}, 2 \mathrm{H}, \mathrm{C}_{\text {cluster }} H\right), 4.50\left(\right.$ br s, $\left.4 \mathrm{H}, \mathrm{C}_{\text {cluster }} H\right)$, 4.04 (s, 2H, Ccluster $H), 3.47$ (s, 12H, N(CH3)4). $1 \mathrm{H}\{11 \mathrm{~B}\} \mathrm{NMR}\left(\mathrm{CD}_{3} \mathrm{COCD}_{3}\right): \delta=4.69$ (s, $2 \mathrm{H}$, Ccluster $H$ ), 4.50 (br s, 4H, Ccluster $H$ ), 4.04 (s, 2H, Ccluster $H), 3.70$ (s, 1H, B-H) 3.47 (s, 12H, $\left.\mathrm{N}\left(\mathrm{CH}_{3}\right) 4\right), 3.05-1.89(\mathrm{~s}, 15 \mathrm{H}, \mathrm{B}-H) .13 \mathrm{C}\{1 \mathrm{H}\} \mathrm{NMR}\left(\mathrm{CD}_{3} \mathrm{COCD}_{3}\right): \delta=59.33$ (C cluster), 55.68 $\left.\left(\mathrm{s}, \mathrm{N}\left(\mathrm{CH}_{3}\right) 4\right)\right),{ }_{11} \mathrm{~B} \mathrm{NMR}\left(\mathrm{CD}_{3} \mathrm{COCD}_{3}\right): \delta=11.2\left(\mathrm{~d},{ }_{1} J(\mathrm{~B}, \mathrm{H})=139\right), 7.8\left(\mathrm{~d},{ }_{1} J(\mathrm{~B}, \mathrm{H})=141\right), 4.1$ $\left(\mathrm{d},{ }_{1} J(\mathrm{~B}, \mathrm{H})=144\right),-0.6\left(\mathrm{~d},{ }_{1} J(\mathrm{~B}, \mathrm{H})=113\right),-1.8\left(\mathrm{~d},{ }_{1} J(\mathrm{~B}, \mathrm{H})=110\right),-2.7\left(\mathrm{~d},{ }_{1} J(\mathrm{~B}, \mathrm{H})=95\right),-3.9$ $\left(\mathrm{d},{ }_{1} J(\mathrm{~B}, \mathrm{H})=135\right),-11.1\left(\mathrm{~d},{ }_{1} J(\mathrm{~B}, \mathrm{H})=191\right),-13.2\left(\mathrm{~d},{ }_{1} J(\mathrm{~B}, \mathrm{H})=86\right),-13.8(\mathrm{~s}, 2 \mathrm{~B}, \mathrm{~B}-\mathrm{I}),-15.1(\mathrm{br}$

s), $-16.2(\mathrm{~d}, 1 J(\mathrm{~B}, \mathrm{H})=110),-21.1\left(\mathrm{~d},{ }_{1} J(\mathrm{~B}, \mathrm{H})=75\right)$. MALDI-TOF MS: $m / z=574.8[\mathrm{M}$, $100 \%]$.

Synthesis of [NMe 4$\left.]\left[3,3 \text { '-Co-(1-Me-9,12-I2-1,2-closo- } \mathrm{C}_{2} \mathrm{~B} 9 \mathrm{H}_{8}\right)_{2}\right]$ To a stirring solution of [HNMe3][7-Me-5,6-I2-7,8-nido- $\left.\mathrm{C}_{2} \mathrm{~B} 9 \mathrm{H} 9\right](120 \mathrm{mg}, 0.25 \mathrm{mmol})$ in THF $(10 \mathrm{ml})$ cooled to 0 
${ }^{\circ} \mathrm{C}$ in an ice-water bath was added, dropwise, a solution of $\mathrm{K} t \mathrm{BuO}$ in $\mathrm{THF}(1.3 \mathrm{ml}, 1 \mathrm{M}$, $1.26 \mathrm{mmol})$. In parallel a $\mathrm{CoCl}_{2}(165 \mathrm{mg}, 01.26 \mathrm{mmol})$ solution in THF $(5 \mathrm{ml})$ was prepared. The solution was then transferred via a syringe over the initial reaction mixture, previously cooled to $0{ }^{\circ} \mathrm{C}$. The resulted mixture was heated to reflux for 6 hours. The solvent was then removed under reduced pressure. $10 \mathrm{ml}$ of diethyl ether and $10 \mathrm{ml}$ of $\mathrm{HCl}(37 \%, 1 \mathrm{M})$ aqueous solution were then added to the residue. The mixture was hardly shaken and the two layers were separated. The organic layer was separated from the mixture and the aqueous layer was extracted with diethyl ether $(3 \times 10 \mathrm{ml})$. The combined organic phase was dried over $\mathrm{MgSO}_{4}$, filtered and the solvent removed under reduce pressure. Afterwards, an aqueous solution of [ $\left.\mathrm{NMe}_{4}\right] \mathrm{Cl}$ was added dropwise until no more precipitate was formed. The orange solid was filtered and rinsed with water to give [NMe4][3,3'-Co(1-Me-9,12-I2-1,2-closo- $\left.\left.\mathrm{C}_{2} \mathrm{~B} 9 \mathrm{H} 8\right)_{2}\right] \quad(165 \mathrm{mg}, \quad 70 \%)$. Elemental analysis for $\mathrm{C}_{10} \mathrm{H}_{34} \mathrm{~B} 18 \mathrm{CoI} 4 \mathrm{~N}$ : calc: C 12.92, H 3.69, N 1.51; found C 12.52, H 3.59, N 1.20. IR (KBr): $v=3020\left(\mathrm{C}_{\mathrm{c}-\mathrm{H}}\right), 2922,2853(\mathrm{Calkyl}-\mathrm{H}), 2577(\mathrm{~B}-\mathrm{H}), 1479\left(\mathrm{~N}-\mathrm{CH}_{3}\right) .{ }_{1} \mathrm{H}\left(\mathrm{CD}_{3} \mathrm{COCD}_{3}\right): \delta=$ $3.45\left(\mathrm{~N}\left(\mathrm{CH}_{3}\right) 4\right), 2.42\left(\mathrm{~s}, 3 \mathrm{H}, \mathrm{C}\right.$ cluster- $\left.\left.\mathrm{CH}_{3}\right), 2.29\left(\mathrm{~s}, 3 \mathrm{H}, \mathrm{C}_{\text {cluster- }} \mathrm{CH}_{3}\right)\right),{ }_{1} \mathrm{H}\{11 \mathrm{~B}\} \quad \mathrm{NMR}$ $\left(\mathrm{CD}_{3} \mathrm{COCD}_{3}\right): \delta=3.76-1.71(\mathrm{~m}, \mathrm{~B}-H), 3.48\left(\mathrm{~s}, 12 \mathrm{H}, \mathrm{N}\left(\mathrm{CH}_{3}\right) 4\right), 2.42(\mathrm{~s}, 3 \mathrm{H}, \mathrm{C}$ cluster-CH3), $\left.2.29\left(\mathrm{~s}, 3 \mathrm{H}, \mathrm{C}_{\text {cluster- }} \mathrm{CH}_{3}\right)\right),{ }_{13} \mathrm{C}\{1 \mathrm{H}\} \quad \mathrm{NMR}\left(\mathrm{CD}_{3} \mathrm{COCD}_{3}\right): \delta=65.47\left(\mathrm{C}_{\text {cluster- }} \mathrm{CH}_{3}\right), 55.52$ $\left(\mathrm{N}\left(\mathrm{CH}_{3}\right) 4\right), 54.13\left(\mathrm{C}_{\text {cluster- }} \mathrm{H}\right), 22.45\left(\mathrm{CH}_{3}\right) .{ }_{11} \mathrm{~B} \mathrm{NMR}\left(\mathrm{CD}_{3} \mathrm{COCD}_{3}\right): \delta=8.9,5.0,-2.7,-4.2$ (m, B-H), -8.8, -12.9, -14.9 (m, B-I). MALDI-TOF MS: $m / z=1117.6$ [M+2I, 47\%], 990.1 [M+I, 100\%], $862.6[\mathrm{M}, 88 \%]$.

\section{Synthesis of [NMe4][3,3'-Co-(1-Ph-9,12-I2-1,2-closo-C2B9H8)2]}

The same procedure as for the obtaining of compound [NMe 4$]\left[3,3^{\prime}-\mathrm{Co}-\left(1-\mathrm{Me}-9,12-\mathrm{I}_{2}-1,2-\right.\right.$ closo- $\left.\left.\mathrm{C}_{2} \mathrm{~B} 9 \mathrm{H}_{8}\right)_{2}\right]$ was followed. The used reactants quantities were: [HNMe3][7-Ph-5,6-I2- 
7,8-nido- $\mathrm{C}_{2} \mathrm{~B} 9 \mathrm{H} 9$ ] (200 mg, $\left.0.39 \mathrm{mmol}\right)$, THF (10 ml), solution of $\mathrm{K} t \mathrm{BuO}$ in THF (1.95 ml, $0.195 \mathrm{mmol}), \mathrm{CoCl}_{2}(254 \mathrm{mg}, 1.95 \mathrm{mmol}), 5 \mathrm{ml}$ of $\mathrm{THF}, 10 \mathrm{ml}$ of $\mathrm{HCl}(37 \%, 0.25 \mathrm{M})$ aqueous solution. The final orange solid compound, [NMe4][3,3'-Co-(1-Ph-9,12-I2-1,2closo- $\left.\left.\mathrm{C}_{2} \mathrm{~B} 9 \mathrm{H} 8\right)_{2}\right]$ was obtained with a yield of $71 \%$ (295 mg). Elemental analysis for $\mathrm{C}_{20} \mathrm{H}_{38} \mathrm{~B} 18 \mathrm{CoI} 4 \mathrm{~N}$ : calc: C 22.80, H 3.64, N 1.33; found C 22.58, H 3.84, N 1.32. IR (KBr): $v=3027\left(\mathrm{C}_{\mathrm{c}-\mathrm{H}}\right), 2951,2923,2854\left(\mathrm{C}\right.$ alkyl-H)$, 2558(\mathrm{~B}-\mathrm{H}), 1479\left(\mathbf{N}-\mathrm{CH}_{3}\right) .{ }_{1} \mathrm{H}$ NMR $\left(\mathrm{CD}_{3} \mathrm{COCD}_{3}\right): \delta=7.66-7.14\left(\mathrm{~m}, 10 \mathrm{H}, \mathrm{C}_{6} \mathrm{H}_{5}\right), 4.63\left(\mathrm{~s}, 2 \mathrm{H}, \mathrm{C}_{\text {cluster- }-\mathrm{H}),} 3.47\left(\mathrm{~N}\left(\mathrm{CH}_{3}\right) 4\right)\right.$. ${ }_{1} \mathrm{H}\{11 \mathrm{~B}\}$ NMR $\left(\mathrm{CD}_{3} \mathrm{COCD}_{3}\right): \delta=7.66-7.14\left(\mathrm{~m}, 10 \mathrm{H}, \mathrm{C}_{6} \mathrm{H}_{5}\right), 4.80-1.53(\mathrm{~m}, \mathrm{~B}-\mathrm{H}), 4.63(\mathrm{~s}$, $\left.2 \mathrm{H}, \mathrm{C}_{\text {cluster }} \mathrm{H}\right), 3.47\left(\mathrm{~N}\left(\mathrm{CH}_{3}\right) 4\right),{ }_{13} \mathrm{C}\{1 \mathrm{H}\}$ NMR $\left(\mathrm{CD}_{3} \mathrm{COCD}_{3}\right): \delta=141.45,140.70\left(\mathrm{C}_{\text {ipso }}\right.$ of $\left.\mathrm{C}_{6} \mathrm{H}_{5}\right), 129.15,128.45,127.97,127.29,125.59,122.88\left(\mathrm{C}_{6} \mathrm{H} 5\right), 71.29$ ( $\left.\mathrm{C}_{\text {cluster- }} \mathrm{C}_{6} \mathrm{H}_{5}\right), 55.46$ $\left(\mathrm{N}\left(\mathrm{CH}_{3}\right) 4\right), 53.94,50.59\left(C_{\text {cluster-H}}\right) .11 \mathrm{~B}$ NMR $\left(\mathrm{CD}_{3} \mathrm{COCD}_{3}\right): \delta=9.4,5.6,-3.7,-9.4(\mathrm{~m}, \mathrm{~B}-$ H), -14.6 (s, B-I). MALDI-TOF MS: $m / z=1115.8$ [M+I, 17\%], 988.2 [M, 100\%], 860.8 [M-I, 17\%].

\section{Synthesis of [NMe4][3,3'-Co-(4,7-I2-1,2-closo-C2B9H9)2]}

The same procedure as for the obtaining of compound [NMe4][3,3'-Co-(1-Me-9,12-I2-1,2closo- $\left.\left.\mathrm{C}_{2} \mathrm{~B} 9 \mathrm{H} 8\right)_{2}\right]$ was followed. The used reactants quantities were: [HNMe3][9,11-I2-7,8nido- $\mathrm{C}_{2} \mathrm{~B}_{9} \mathrm{H}_{10}$ ] (200 mg, $\left.0.63 \mathrm{mmol}\right)$, THF (20 ml), solution of $\mathrm{K} t \mathrm{BuO}$ in THF (3.8ml, $1 \mathrm{M}$, $3.8 \mathrm{mmol}), \mathrm{CoCl}_{2}(656 \mathrm{mg}, 5.05 \mathrm{mmol}), 10 \mathrm{ml}$ of $\mathrm{THF}, 10 \mathrm{ml}$ of $\mathrm{HCl}(37 \%, 0.25 \mathrm{M})$ aqueous solution. The final orange solid compound, [NMe4][3,3'-Co-(4,7-I2-1,2-closo-C2 2 B9H9)2] was obtained with a yield of $57 \% \quad(350 \mathrm{mg})$, after purification on silica gel column chromatography, using $\mathrm{CH}_{2} \mathrm{Cl}_{2}$ for elution. Elemental analysis for $\mathrm{C}_{8} \mathrm{H}_{30} \mathrm{~B}_{18} \mathrm{CoI} 4 \mathrm{~N}$ : calc: $\mathrm{C}$ 10.66, H 3.35, N 1.55; found C 10.76, H 3.21, N 1.43. IR (ATR): v=3018 (s, vs(Ccluster-H)), 2955, 2922, 2858 (s, vs $($ Calkyl-H $), 2564$ (vs, vs $(B-H)), 1478\left(v_{s}(\mathbf{N}-\mathbf{C H} 3)\right) .1 \mathrm{H}$ NMR 
$\left(\mathrm{CD}_{3} \mathrm{COCD}_{3}\right): \delta=4.83\left(\mathrm{~s}, 2 \mathrm{H}, \mathrm{C}_{\text {cluster }} H\right), 4.73(\mathrm{~s}, 2 \mathrm{H}, \mathrm{C}$ cluster $H), 3.47\left(\mathrm{~s}, 12 \mathrm{H}, \mathrm{N}\left(\mathrm{CH}_{3}\right) 4\right)$.

${ }_{1} \mathrm{H}\{11 \mathrm{~B}\}$ NMR $\left(\mathrm{CD}_{3} \mathrm{COCD}_{3}\right): \delta=4.83\left(\mathrm{~s}, 2 \mathrm{H}, \mathrm{C}_{\text {cluster }} H\right), 4.73(\mathrm{~s}, 2 \mathrm{H}, \mathrm{C}$ cluster $H), 3.47(\mathrm{~s}, 12 \mathrm{H}$, $\left.\mathrm{N}\left(\mathrm{CH} H_{3}\right) 4\right), 3.06-2.30$ (s, $\left.14 \mathrm{H}, \mathrm{B}-H\right) .{ }_{13} \mathrm{C}\{1 \mathrm{H}\}$ NMR ( $\left.\mathrm{CD}_{3} \mathrm{COCD}_{3}\right): \delta=63.17$ (Ccluster), 55.25 $\left.\left(\mathrm{s}, \mathrm{N}\left(\mathrm{CH}_{3}\right) 4\right)\right) .{ }_{11} \mathrm{~B}$ NMR $\left(\mathrm{CD}_{3} \mathrm{COCD}_{3}\right): \delta=14.9\left(\mathrm{~d},{ }_{1} J(\mathrm{~B}, \mathrm{H})=145\right), 4.7\left(\mathrm{~d},{ }_{1} J(\mathrm{~B}, \mathrm{H})=137\right), 3.4$ $(\mathrm{d}, 1 J(\mathrm{~B}, \mathrm{H})=138),-0.8\left(\mathrm{~d},{ }_{1} J(\mathrm{~B}, \mathrm{H})=147\right),-9.1(\mathrm{~d}, \mathrm{~B}-\mathrm{H}+2 \mathrm{~B}-\mathrm{I}),-11.4(\mathrm{~d}, \mathrm{~B}-\mathrm{H}),-12.9(\mathrm{~s}, 2 \mathrm{~B}-$ I), $-20.6\left(\mathrm{~d},{ }_{1} J(\mathrm{~B}, \mathrm{H})=162\right)$. MALDI-TOF MS: $m / z=827.9$ [M, 100\%], 702 [M-1, 39\%], 576 [M-2I, 27\%].

\section{Synthesis of [NMe4] [3,3'-Co-(1-Me-8,9,10,12-I4-1,2-closo-C2B9H6)2]}

The same procedure as for the obtaining of compound [NMe4][3,3'-Co-(1-Me-9,12-I2-1,2closo- $\left.\mathrm{C}_{2} \mathrm{~B} 9 \mathrm{H}_{8}\right)_{2}$ ] was followed. The used reactants quantities were: [HNMe3][7-Me1,5,6,10-I4-7,8-nido- $\left.\mathrm{C}_{2} \mathrm{~B} 9 \mathrm{H} 7\right]$ ( $\left.60 \mathrm{mg}, 0.08 \mathrm{mmol}\right)$, THF ( $\left.5 \mathrm{ml}\right)$, solution of butyllithium in hexane $(0.105 \mathrm{ml}, 1.6 \mathrm{M}, 0.168 \mathrm{mmol}), \mathrm{CoCl}_{2}(32.8 \mathrm{~g}, 0.253 \mathrm{mmol}), 10 \mathrm{ml}$ of diethyl ether, $10 \mathrm{ml}$ of $\mathrm{HCl}(37 \%, 0.25 \mathrm{M})$ aqueous solution. The final orange solid compound, [NMe4][3,3'-Co(1-Me-8,9,10,12-I4-1,2-closo- $\left.\left.\mathrm{C}_{2} \mathrm{~B}_{9} \mathrm{H}_{6}\right)_{2}\right]$, was obtained with a yield of $65 \%$ (64 mg). Elemental analysis for $\mathrm{C}_{10} \mathrm{H}_{30} \mathrm{~B} 18 \mathrm{Col} 8 \mathrm{~N}$ : calc: C 8.38, H 2.11, N 0.98; found $\mathrm{C}$ 8.21, H 2.36, N 1.04. IR (KBr): v= $3022(\mathrm{w}, v(\mathrm{C}$ cluster-H)), 2952, 2924, 2853 (s, v(Ccluster$\left.\left.\mathrm{CH}_{3}\right),\left(\mathrm{N}\left(\mathrm{CH}_{3}\right) 4\right)\right), 2590(\mathrm{~s}, v(\mathrm{~B}-\mathrm{H})) .{ }_{1} \mathrm{H}$ NMR $\left(\mathrm{CD}_{3} \mathrm{COCD}_{3}\right): \delta=5.18(\mathrm{~s}, 2 \mathrm{H}, \mathrm{C}$ cluster-H), $3.45\left(\mathrm{~s}, 12 \mathrm{H}, \mathrm{N}\left(\mathrm{CH}_{3}\right) 4\right), 2.55\left(\mathrm{~s}, 3 \mathrm{H}, \mathrm{C}_{\text {cluster- }} \mathrm{CH}_{3}\right) .1 \mathrm{H}\{11 \mathrm{~B}\} \mathrm{NMR}\left(\mathrm{CD}_{3} \mathrm{COCD}_{3}\right): \delta=5.18(\mathrm{~s}$, 2H, Ccluster-H), 4.25 (s, B-H), 3.45 (s, 12H, N(CH3)4), 2,93 (s, B-H), 2.84 (s, 3H, Ccluster$\left.\mathrm{CH}_{3}\right), 2.55$ (s, $\left.3 \mathrm{H}, \mathrm{C}_{\text {cluster- }} \mathrm{CH}_{3}\right) .{ }_{13} \mathrm{C}\{1 \mathrm{H}\} \mathrm{NMR}\left(\mathrm{CD}_{3} \mathrm{COCD}_{3}\right): \delta=69.77$ (Ccluster- $\left.\mathrm{CH}_{3}\right), 60.66$ (Ccluster-H), $55.36\left(\mathrm{~N}\left(\mathrm{CH}_{3}\right) 4\right), 22.42\left(\mathrm{CH}_{3}\right),{ }_{11} \mathrm{~B} \mathrm{NMR}\left(\mathrm{CD}_{3} \mathrm{COCD}_{3}\right): \delta=-0.5,-1.3,-3.2,-4.9$ (m, B-H), -7.4, -9.4 (m, 4B, B-I), -11.7, -13.2 (m, B-H). MALDI-TOF-MS: m/z= 1363 [M, 27\%], 1237 [M-I, 10\%], 837 [M-4I, 100\%]. 


\section{Synthesis of [NMe4][3,3'-Co-(1-Ph-8,9,10,12-I4-1,2-closo-C2B9H6)2]}

The same procedure as for the obtaining of compound [NMe4][3,3'-Co-(1-Me-9,12-I2-1,2closo- $\left.\left.\mathrm{C}_{2} \mathrm{~B}_{9} \mathrm{H}_{8}\right)_{2}\right]$ was followed. The used reactants quantities were: [HNMe3][7-Ph-1,5,6,10I4-7,8-nido- $\left.\mathrm{C}_{2} \mathrm{~B}_{9} \mathrm{H}_{7}\right]$ (100 $\left.\mathrm{mg}, 0.12 \mathrm{mmol}\right)$, THF $(5 \mathrm{ml})$, solution of butyllithium in hexane (0.16ml, 1.6M, $0.258 \mathrm{mmol}), \mathrm{CoCl}_{2}(50.2 \mathrm{~g}, 0.387 \mathrm{mmol}), 10 \mathrm{ml}$ of diethyl ether, $10 \mathrm{ml}$ of $\mathrm{HCl}(37 \%, 0.25 \mathrm{M})$ aqueous solution. The final orange solid compound, [NMe4][3,3'-Co(1$\mathrm{Ph}-8,9,10,12-\mathrm{I}_{4}-1,2-$ closo- $\left.\left.\mathrm{C}_{2} \mathrm{~B}_{9} \mathrm{H}_{6}\right)_{2}\right]$, was obtained with a yield of $69 \%$ (42 $\mathrm{mg}$ ). Elemental analysis for $\mathrm{C}_{20} \mathrm{H}_{34} \mathrm{~B} 18 \mathrm{CoI} 8 \mathrm{~N}$ : calc: C 15.43, H 2.20, N 0.90; found $\mathrm{C} 15.27, \mathrm{H} 2.17, \mathrm{~N} 0.87$. IR (KBr): v= $3030\left(\mathrm{w}, \mathrm{v}(\mathrm{C}\right.$ cluster-H) $), 2952,2923,2853\left(\mathrm{~s}, \mathrm{v}\left(\mathrm{N}\left(\mathrm{CH}_{3}\right) 4\right), 2606(\mathrm{~s}, \mathrm{v}(\mathrm{B}-\mathrm{H}) .1 \mathrm{H}\right.$ NMR (CD $\left.{ }_{3} \mathrm{COCD}_{3}\right): \delta=7.26-6.98\left(\mathrm{~m}, 10 \mathrm{H}, \mathrm{C}_{6} \mathrm{H} 5\right), 5.71\left(\mathrm{~s}, 2 \mathrm{H}, \mathrm{C}_{\text {cluster-H}}\right), 3.45(\mathrm{~s}, 12 \mathrm{H}$, $\left.\mathrm{N}\left(\mathrm{CH}_{3}\right) 4\right) .{ }_{1} \mathrm{H}\left\{{ }_{11} \mathrm{~B}\right\} \mathrm{NMR}\left(\mathrm{CD}_{3} \mathrm{COCD}_{3}\right): \delta=7.26-6.98(\mathrm{~m}, 10 \mathrm{H}, \mathrm{C} 6 \mathrm{H} 5), 5.71\left(\mathrm{~s}, 2 \mathrm{H}, \mathrm{C}_{\text {cluster- }}\right.$ H), 4.50 (s, B-H), $2.63(\mathrm{~s}, \mathrm{~B}-\mathrm{H}), 2.41(\mathrm{~s}, \mathrm{~B}-\mathrm{H}), 3.45\left(\mathrm{~s}, 12 \mathrm{H}, \mathrm{N}\left(\mathrm{CH}_{3}\right) 4\right) .{ }_{13} \mathrm{C}\left\{{ }_{1} \mathrm{H}\right\} \mathrm{NMR}$ $\left(\mathrm{CD}_{3} \mathrm{COCD}_{3}\right): \delta=137.40\left(\mathrm{C}_{\mathrm{ipso}}\right.$ of $\left.\mathrm{C}_{6} \mathrm{H}_{5}\right), 129.93,128.73,128.12,127.03,126.63\left(\mathrm{C}_{6} \mathrm{H}_{5}\right)$ $64.01\left(\mathrm{C}_{\text {cluster-H}} \mathrm{H}\right), 55.29\left(\mathrm{~N}\left(\mathrm{CH}_{3}\right) 4\right) .11 \mathrm{~B}$ NMR $\left(\mathrm{CD}_{3} \mathrm{COCD}_{3}\right): \delta=-1.89,-6.70,-11.30,-14.02$ (m, 18B, B-H + B-I). MALDI-TOF-MS: m/z= 1486 [M, 17\%], 1359 [M-I, 33\%], 1232 [M2I, 20\%], 1104 [M-3I, 12\%], 978 [M-4I, 16\%], 840 [M-5I, 32\%], 713 [M-6I, 100\%].

\section{Synthesis of [NMe4][3,3'-Co-(4,9,12-I3-1,2-closo-C2 $\left.\left.2 \mathrm{~B}_{9} \mathrm{H} 8\right)_{2}\right]$}

To a stirring solution of [HNMe $]\left[5,6,9-\mathrm{I}_{3}-7,8-\right.$ nido- $\left.\mathrm{C}_{2} \mathrm{~B}_{9} \mathrm{H}_{9}\right](90 \mathrm{mg}, 0.15 \mathrm{mmol})$ in THF (3 $\mathrm{mL}$ ) was added, dropwise, a solution of potassium $\mathrm{K} t \mathrm{BuO}$ in $\mathrm{THF}(0.94 \mathrm{~mL}, 1 \mathrm{M}$, $0.94 \mathrm{mmol})$. After 50 minute, the resulting solution was then transferred via a syringe onto a $\mathrm{CoCl}_{2}$ anhydrous solution $(122 \mathrm{mg}, 0.94 \mathrm{mmol})$ in THF $(5 \mathrm{~mL})$, following which the reaction was heated to reflux overnight. After removal of the solvent, $10 \mathrm{~mL}$ of diethyl ether was added. This solution was extracted three times with $10 \mathrm{~mL}$ of diluted $\mathrm{HCl}(37 \%$, 
0.25M). The organic layer was separated and dried over anhydrous MgSO4. The solvent was removed, the product was redissolved in the minimum volume of water and an aqueous solution containing an excess of $\left[\mathrm{NMe}_{4}\right] \mathrm{Cl}$ was added to precipitate the product. The precipitate was collected by filtration and dried in vacuum. The final orange solid [NMe $]\left[3,3\right.$ '-Co-(4,9,12-I3-1,2-closo- $\left.\left.\mathrm{C}_{2} \mathrm{~B}_{9} \mathrm{H}_{8}\right) 2\right]$, was obtained with a yield of $83 \%$ (76 mg). Elemental analysis for $\mathrm{C}_{8} \mathrm{H}_{28} \mathrm{~B}_{18} \mathrm{CoI}_{6} \mathrm{~N}$ : calc: C 8.33, H 2.45, N 1.21; found C 8.41, H 2.26, N 1.23. IR (KBr): v= 3019 (s, v(Ccluster-H)), 2947, 2918, 2855 (Calkyl-H), 2589 (s, vs(B-H)), $1477\left(v \mathrm{~s}\left(\mathrm{~N}-\mathrm{CH}_{3}\right)\right), 941\left(\mathrm{~s}, \operatorname{vas}\left(\mathrm{CH}_{3}\right)\right)$. $1 \mathrm{HNMR}\left(\mathrm{CD}_{3} \mathrm{COCD}_{3}\right): \delta=5.8(\mathrm{br} \mathrm{s}, \mathrm{C}$ cluster-H), 5.2 (s, Ccluster-H), 5.0(s, C cluster-H), 4.5(s, C cluster-H) $3.46\left(\mathrm{~s}, 12 \mathrm{H}, \mathrm{N}\left(\mathrm{CH}_{3}\right) 4\right),{ }_{1} \mathrm{H}\{11 \mathrm{~B}\}$ NMR $\left(\mathrm{CD}_{3} \mathrm{COCD}_{3}\right): \delta=5.8\left(\right.$ br s, Ccluster-H), $5.2\left(\mathrm{~s}, \mathrm{C}_{\text {cluster-H}} \mathrm{H}\right), 5.0\left(\mathrm{~s}, \mathrm{C}_{\text {cluster}}-\mathrm{H}\right), 4.5\left(\mathrm{~s}, \mathrm{C}_{\text {cluster-H}}\right)$, 4.4, 3.9, 3.8, 2.6, 2.5, 2.0 (br s, B-H), 3.46 (s, 12H, N(CH3)4). ${ }_{13} \mathrm{C}\left\{{ }_{1} \mathrm{H}\right\}$ NMR $\left(\mathrm{CD}_{3} \mathrm{COCD}_{3}\right)$ : 59.0, 56.9 (s, Ccluster-H), 55.3(s, $\left.\left(\mathrm{CH}_{3}\right) 4\right)$ 11B NMR (CD3COCD 3$): \delta=12.0,7.5$ and $-1.1(\mathrm{br}$ s, 6B, B-H + B-I), -10.0,-10.7, -13.4, -14.1, -19.2 (br s, 12B, B-H + B-I). MALDI-TOF-MS: $\mathrm{m} / \mathrm{z}=695.7[\mathrm{M}-3 \mathrm{I}, 46 \%$ ], 953.8[M-I, 15\%] 1079.8 [M, 100\%], 1205.7[M+I, 15\%].

\section{Synthesis of [NMe4][3,3'-Co-(9,12-Me2-1,2-closo-C2B9H9)2]}

A solution of [ $\left.\mathrm{NMe}_{4}\right]\left[3,3-\mathrm{Co}\left(9,12-\mathrm{I}_{2}-1,2-\right.\right.$ closo $\left.\left._{-} \mathrm{C}_{2} \mathrm{~B} 9 \mathrm{H}_{9}\right) 2\right]-(300 \mathrm{mg}, 0.33 \mathrm{mmol})$ in THF (40 $\mathrm{mL})$ was treated with methylmagnesium bromide $(1.1 \mathrm{~mL}, 3.0 \mathrm{M}$ in diethyl ether; 3.32 $\mathrm{mmol})$ at $-84^{\circ} \mathrm{C}$, forming a brown precipitate. The mixture was allowed to warm to room temperature and then $\left[\mathrm{PdCl}_{2}\left(\mathrm{PPh}_{3}\right)_{2}\right](28.5 \mathrm{mg}, 0.04 \mathrm{mmol})$ and $\mathrm{CuI}(9.57 \mathrm{mg}, 0.05 \mathrm{mmol})$ were added. The mixture was refluxed $15 \mathrm{~h}$. Twenty drops of water were then added to quench the excess Grignard reagent, and the solvent was removed in vacuum. The residue was extracted with diethyl ether, leaving some dark material, which was discarded and the combined organic layers were dried over anhydrous magnesium sulfate. The solvent was 
removed, the product was redissolved in the minimum volume of ethanol and an aqueous solution containing an excess of $\left[\mathrm{NMe}_{4}\right] \mathrm{Cl}$ was added to precipitate the product. The precipitate was collected by filtration and dried in vacuum. The solid was subjected to flash silica gel chromatography using the next solvent mixture methylene chloride:acetonitrile 7:4. The final orange solid compound, [NMe4][3,3-Co(9,12-Me2-1,2-closo- $\left.\left.\mathrm{C}_{2} \mathrm{~B} 9 \mathrm{H}_{9}\right) 2\right]$, was obtained with a yield of $77 \%(120 \mathrm{mg})$. Elemental analysis for $\mathrm{C}_{12} \mathrm{H}_{42} \mathrm{~B} 18 \mathrm{CoN}$ : calc: $\mathrm{C}$ 31.75, H 9.32, N 3.09; found $\mathrm{C} 32.66, \mathrm{H}$ 8.63, N 3.23. IR (KBr): $v=3040,3029(\mathrm{~s}$, $v\left(\right.$ cluster-H)), 2933, 2897, $2830\left(\right.$ Calkyl-H), 2541, $2516\left(s, v_{s}(\mathrm{~B}-\mathrm{H})\right), 1481\left(v_{\mathrm{s}}\left(\mathrm{N}-\mathrm{CH}_{3}\right)\right), 945$ (s, vas $\left.\left(\mathrm{CH}_{3}\right)\right)$. $1 \mathrm{HNMR}\left(\mathrm{CD}_{3} \mathrm{COCD}_{3}\right): \delta=3.75(\mathrm{~s}, 4 \mathrm{H}, \mathrm{C}$ cluster- $\mathrm{H}), 3.47\left(\mathrm{~s}, 12 \mathrm{H}, \mathrm{N}\left(\mathrm{CH}_{3}\right) 4\right)$, $0.08\left(\mathrm{~s}, 12 \mathrm{H}, \mathrm{BCH}_{3}\right) ; 1 \mathrm{H}\{11 \mathrm{~B}\} \mathrm{NMR}\left(\mathrm{CD}_{3} \mathrm{COCD}_{3}\right): \delta=3.75(\mathrm{~s}, 4 \mathrm{H}, \mathrm{C}$ cluster-H), $3.47(\mathrm{~s}, 12 \mathrm{H}$, $\left.\mathrm{N}\left(\mathrm{CH}_{3}\right) 4\right), 3.17$ (br s, 2H, B-H), 2.77 (br s, 2H, B-H), 2.66 ( br s, 2H, B-H), 2.64 ( br s, 2H, B-H), 1.53 (br s, 6H, B-H), $0.08\left(\mathrm{~s}, 12 \mathrm{H}, \mathrm{BCH}_{3}\right) ; 13 \mathrm{C}\{1 \mathrm{H}\} \mathrm{NMR}\left(\mathrm{CD}_{3} \mathrm{COCD}_{3}\right): \delta=55.7(\mathrm{~s}$, $\left.\left(\mathrm{CH}_{3}\right)_{4}\right) 45.9$ (s, Ccluster-H), 5.07-2.57 (br m, B-CH3). ${ }_{11} \mathrm{~B}$ NMR $\left(\mathrm{CD}_{3} \mathrm{COCD}_{3}\right): \delta=10.9(\mathrm{~d}$, $\left.{ }_{1} \mathrm{~J}(\mathrm{~B}, \mathrm{H})=137,2 \mathrm{~B}, \mathrm{~B}\left(8,8^{\prime}\right)\right), 4.1\left((4 \mathrm{BC}+2 \mathrm{BH}), \mathrm{B}\left(9,9^{\prime}, 10,10^{\prime}, 12,12^{\prime}\right),-3.7\left(\mathrm{~d},{ }_{1} \mathrm{~J}(\mathrm{~B}, \mathrm{H})=\right.\right.$ 146, 4B, B(4,4',7,7')), -16.9 (d, $\left.1 \mathrm{~J}(\mathrm{~B}, \mathrm{H})=148,4 \mathrm{~B}, \mathrm{~B}\left(5,5^{\prime}, 11,1^{\prime}\right)\right),-22.1\left(\mathrm{~d},{ }_{1} \mathrm{~J}(\mathrm{~B}, \mathrm{H})\right.$ $\left.=164,2 \mathrm{~B}, \mathrm{~B}\left(6,6^{\prime}\right)\right)$. MALDI-TOF-MS: m/z= $379 \cdot 6[\mathrm{M}, 100 \%]$.

\section{Synthesis of [NMe4][3,3'-Co-(8,9,10,12-Me4-1,2-closo-C2 $\left.\left.2 \mathrm{~B}_{9} \mathrm{H}_{7}\right) 2\right]$}

To a stirring solution of $[\mathrm{NBu} 4]\left[1,5,6,10-\mathrm{Me} 4-7,8-\right.$-nido- $\left.\mathrm{C}_{2} \mathrm{~B} 9 \mathrm{H} 8\right]$ (100mg, $\left.0.23 \mathrm{mmol}\right)$ in THF $(6 \mathrm{~mL})$ was added, dropwise, a solution of potassium $\mathrm{K} t \mathrm{BuO}$ in THF $(1.38 \mathrm{~mL}, 1 \mathrm{M}$, $1.38 \mathrm{mmol})$. After 20 minute, the resulting solution was then transferred via a syringe onto a $\mathrm{CoCl}_{2}$ anhydrous solution $(180 \mathrm{mg}, 1.38 \mathrm{mmol})$ in THF $(5 \mathrm{~mL})$, following which the reaction was heated to reflux for $7 \mathrm{~h}$. After removal of the solvent, $10 \mathrm{~mL}$ of diethyl ether was added. This solution was extracted three times with $10 \mathrm{~mL}$ of diluted $\mathrm{HCl}(37 \%$, 
$0.25 \mathrm{M})$. The organic layer was separated and dried over anhydrous $\mathrm{MgSO}_{4}$. The solvent was removed and the orange solid was flash-chromatographed over silica gel using ethyl acetate as eluent. The final orange solid compound, [NBu4][3,3-Co(8,9,10,12-Me4-1,2closo- $\left.\mathrm{C}_{2} \mathrm{~B}_{9} \mathrm{H}_{7}\right)_{2}$ ], was obtained with a yield of $60 \%(47 \mathrm{mg}) . \mathrm{IR}(\mathrm{KBr}): \mathrm{v}=2955,2925$, $2855\left(\mathrm{C}\right.$ alkyl-H), 2598, 2557, $2531\left(\mathrm{~s}, v_{\mathrm{s}}(\mathrm{B}-\mathrm{H})\right) .{ }_{1} \mathrm{HNMR}\left(\mathrm{CD}_{3} \mathrm{Cl}\right): \delta=4.00(\mathrm{~s}, 4 \mathrm{H}, \mathrm{C}$ cluster-H), $3.16\left(\mathrm{t}, \quad 3 \mathrm{~J}(\mathrm{H}, \mathrm{H})=6, \quad 8 \mathrm{H}, \quad\left(\mathrm{CH}_{3} \mathrm{CH}_{2} \mathrm{CH}_{2} \mathrm{CH}_{2}\right) 4 \mathrm{~N}\right), \quad 1.63 \quad(\mathrm{~m}, \quad 3 \mathrm{~J}(\mathrm{H}, \mathrm{H})=9, \quad 8 \mathrm{H}$, $\left.\left(\mathrm{CH}_{3} \mathrm{CH}_{2} \mathrm{CH}_{2} \mathrm{CH}_{2}\right)_{4} \mathrm{~N}\right), 1.48\left(\mathrm{~m},{ }_{3} \mathrm{~J}(\mathrm{H}, \mathrm{H})=6,8 \mathrm{H},\left(\mathrm{CH}_{3} \mathrm{CH}_{2} \mathrm{CH}_{2} \mathrm{CH}_{2}\right){ }_{4} \mathrm{~N}\right), 1.07\left(\mathrm{t},{ }_{3} \mathrm{~J}(\mathrm{H}, \mathrm{H})=\right.$ 6, 12H, $\left.\left(\mathrm{CH}_{3} \mathrm{CH}_{2} \mathrm{CH}_{2} \mathrm{CH}_{2}\right) 4 \mathrm{~N}\right), 0.09\left(\mathrm{~s}, 6 \mathrm{H}, \mathrm{CH}_{3}\right), 0.05\left(\mathrm{~s}, 6 \mathrm{H}, \mathrm{CH}_{3}\right),-0.19\left(\mathrm{~s}, 12 \mathrm{H}, \mathrm{CH}_{3}\right)$. ${ }_{1} \mathrm{H}\left\{{ }_{11 \mathrm{~B}}\right\} \quad \mathrm{NMR}\left(\mathrm{CD}{ }_{3} \mathrm{Cl}\right): \quad \delta=4.00\left(\mathrm{~s}, 4 \mathrm{H}, \mathrm{C}_{\text {cluster }-\mathrm{H}),} 3.16(\mathrm{t}, 3 \mathrm{~J}(\mathrm{H}, \mathrm{H})=6,8 \mathrm{H}\right.$, $\left.\left(\mathrm{CH}_{3} \mathrm{CH}_{2} \mathrm{CH}_{2} \mathrm{CH}_{2}\right)_{4} \mathrm{~N}\right), 2.27$ (br s, B-H), $1.63\left(\mathrm{~m},{ }_{3} \mathrm{~J}(\mathrm{H}, \mathrm{H})=9,8 \mathrm{H},\left(\mathrm{CH}_{3} \mathrm{CH}_{2} \mathrm{CH}_{2} \mathrm{CH}_{2}\right){ }_{4} \mathrm{~N}\right)$, $1.48\left(\mathrm{~m}, \quad{ }_{3} \mathrm{~J}(\mathrm{H}, \mathrm{H})=6, \quad 8 \mathrm{H}, \quad\left(\mathrm{CH}_{3} \mathrm{CH}_{2} \mathrm{CH}_{2} \mathrm{CH}_{2}\right) 4 \mathrm{~N}\right), \quad 1.07 \quad(\mathrm{t}, \quad 3 \mathrm{~J}(\mathrm{H}, \mathrm{H})=6, \quad 12 \mathrm{H}$, $\left.\left(\mathrm{CH}_{3} \mathrm{CH}_{2} \mathrm{CH}_{2} \mathrm{CH}_{2}\right) 4 \mathrm{~N}\right), 0.09\left(\mathrm{~s}, 6 \mathrm{H}, \mathrm{CH}_{3}\right), 0.05\left(\mathrm{~s}, 6 \mathrm{H}, \mathrm{CH}_{3}\right),-0.19\left(\mathrm{~s}, 12 \mathrm{H}, \mathrm{CH}_{3}\right) . \quad{ }_{11} \mathrm{~B}$ NMR $\left(\mathrm{CD}_{3} \mathrm{Cl}\right): \delta=12.8\left(\mathrm{~s}, 2 \mathrm{~B}, \mathrm{~B}-\mathrm{CH}_{3}\right), 9.9\left(\mathrm{~s}, 2 \mathrm{~B}, \mathrm{~B}-\mathrm{CH}_{3}\right), 1.9\left(\mathrm{~s}, 4 \mathrm{~B}, \mathrm{~B}-\mathrm{CH}_{3}\right),-5.0(\mathrm{~d}$, $\left.{ }_{1} J(\mathrm{~B}, \mathrm{H})=138\right),-17.2\left(\mathrm{~d},{ }_{1} J(\mathrm{~B}, \mathrm{H})=135\right),-24.0\left(\mathrm{~d},{ }_{1} J(\mathrm{~B}, \mathrm{H})=137\right)$. MALDI-TOF-MS: $\mathrm{m} / \mathrm{z}=$ 422.4 [M-1Me, 43.7\%], 436.4 [M, 100\%], 450.4 [M+1Me, 51.5\%].

\section{Synthesis of $\mathrm{Na}[\mathrm{NMe} 4]\left[3,3\right.$ '-Co- $\left.\left(8,9,10,12-\mathrm{I}_{4}-1,2-\text { closo- }_{2} \mathrm{C}_{2} \mathrm{~B}_{9} \mathrm{H}_{7}\right)_{2}\right] \cdot 2.5 \mathrm{H}_{2} \mathrm{O}$}

A freshly prepared solution of $\mathrm{Na}\left[\mathrm{C}_{10} \mathrm{H} 8\right](35 \mathrm{mg}, 1.52 \mathrm{mmol})$ in $\mathrm{THF}(10 \mathrm{ml})$ was added over the $\left[\mathrm{NMe}_{4}\right]+$ salt of $\left[3,3^{\prime}-\mathrm{Co}-\left(8,9,10,12-\mathrm{I}_{4}-1,2-\right.\right.$ closo- $\left.\left.\mathrm{C}_{2} \mathrm{~B}_{9} \mathrm{H}_{7}\right) 2\right]-(200 \mathrm{mg}, 0.14 \mathrm{mmol})$ dissolved in THF $(10 \mathrm{ml})$. The mixture was stirred at room temperature for $30 \mathrm{~min}$. Next, the formed grey suspension was removed by filtration. After stripping off the solvent from the filtrate under reduced pressure, a brown solid powder was obtained. Naphthalene was removed by vacuum sublimation to give $\left[3,3^{\prime}-\mathrm{Co}-\left(8,9,10,12-\mathrm{I}_{4}-1,2-\right.\right.$ closo $\left.\left.^{-} \mathrm{C}_{2} \mathrm{~B} 9 \mathrm{H}_{7}\right) 2\right] 2-(180$ mg). Elemental analysis for $\mathrm{C}_{8} \mathrm{H}_{26} \mathrm{~B}_{18} \mathrm{CoI}_{8} \mathrm{NNa} \cdot 2.5 \mathrm{H}_{2} \mathrm{O}$ : calc: $\mathrm{C} 6.51, \mathrm{H} 2.10$, N 0.96; found 
C 6.42, H 2.14, N 0.94. 11B NMR ( $\left.\mathrm{CD}_{3} \mathrm{COCD}_{3}\right): \delta=28.4$ (s, 2B), 20.7 (s, 6B), -55.1 (s, 4B), -95.7 (s, 6B). $1 \mathrm{H}\{11 \mathrm{~B}\}$ NMR $\left(\mathrm{CD}_{3} \mathrm{COCD}_{3}\right): \delta=+45.98($ br s, $\mathrm{C}$ cluster- $H), 3.45\left(\mathrm{~s}, \mathrm{~N}\left(\mathrm{CH}_{3}\right) 4\right),-$ $15.85(\mathrm{~s}, \mathrm{~B}-H)$. IR (KBr): v= $3396(\mathrm{O}-\mathrm{H}), 3025(\mathrm{Cc}-\mathrm{H}), 2923,2853(\mathrm{C}$ alkyl-H), 2587, 2535 (B-H), 1660, 1596 (H-O-H), 1475, 944 (N-C).

\section{Electron paramagnetic resonance spectra (EPR) of I8-[1]2-in $\mathrm{H}_{2} \mathrm{O}$ at $130 \mathrm{~K}(33 \mathrm{mM})$.}

EPR spectra were obtained in an X-Band Bruker ELEXYS E500 spectrometer equipped with a TE102 microwave cavity, a Bruker variable temperature unit and a field frequency lock system Bruker ER $033 \mathrm{M}$. The signal to noise ratio of spectra was increased by accumulation of scans using the F/F lock accessory to guarantee large field reproducibility. Line positions were determined with an NMR Gaussmeter Bruker ER $035 \mathrm{M}$. The modulation amplitude was kept well below the line width, and the microwave power was well below saturation.

\section{Computational Details}

Calculations were performed using the Gaussian09 (revisión D01)[32] with the hybrid B3LYP functional[33] together with LANL2DZ basis set (Hay-Wadt effective core potentials for the iodine and cobalt atoms[34]). In order to improve the convergence of the $\mathrm{Co} 2+$ systems, the option of switching quadratic convergence algorithm and direct inversion in the iterative subspace (DIIS) method was employed. In the calculation of the redox potentials, in order to circumvent the problems to perform the calculation of the thermodynamic magnitudes (free energy) including solvent effects (polarizable conductor calculation model) a thermodynamic cycle was employed.[35] Thus, the free energy to calculate the redox potentials was estimated using the gas phase free energy difference 
between the oxidized and reduced species, while the solvation energies were calculated as the energy difference between the solvated and non-solvated molecules.

\section{Acknowledgments}

The authors are grateful to the following organizations for financial support: the Ministerio de Ciencia e Innovación (MICINN, project numbers CTQ2011-23862-C02-01 and CTQ201344670-R), the Generalitat de Catalunya (projects number 2014SGR149). M. Lupu thanks MICINN for FPU grant. M. Lupu and A. Zaulet are enrolled in the UAB PhD program. 
Chart 1.- Icosahedral dianionic $\left[7,8-\right.$ nido- $\left.\mathrm{C}_{2} \mathrm{~B} 9 \mathrm{H}_{11}\right] 2$ - ligand and pristine monoanionic cobaltabisdicarbollide [3,3'-Co(1,2-closo- $\left.\left.\mathrm{C}_{2} \mathrm{~B}_{9} \mathrm{H}_{11}\right)_{2}\right]$ - with their vertex numbering.
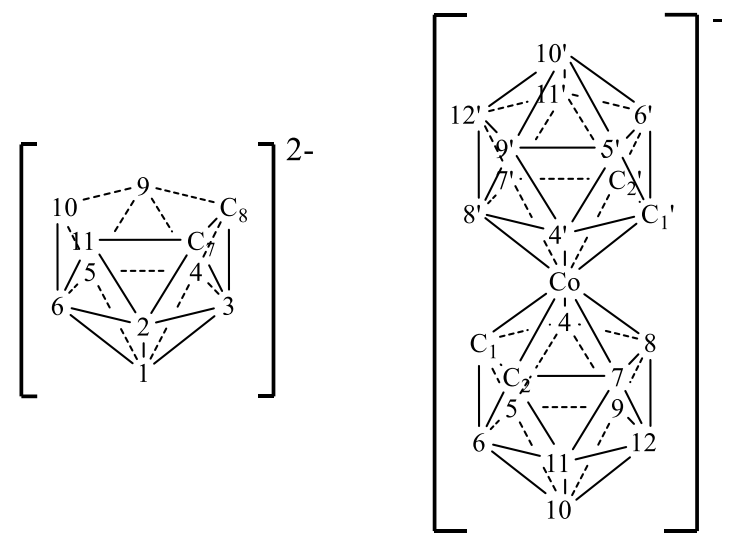
Scheme 1.- Syntheses of [3,3'-Co(9,12-I2-1,2-closo- $\left.\left.\mathrm{C}_{2} \mathrm{~B} 9 \mathrm{H} 9\right)_{2}\right]-\left(\mathrm{I} 4-[1]^{-}\right)$and [3,3'-Co(9,12(Me)2-1,2-closo-C2B9H9)2]-(Me4-[1]-).
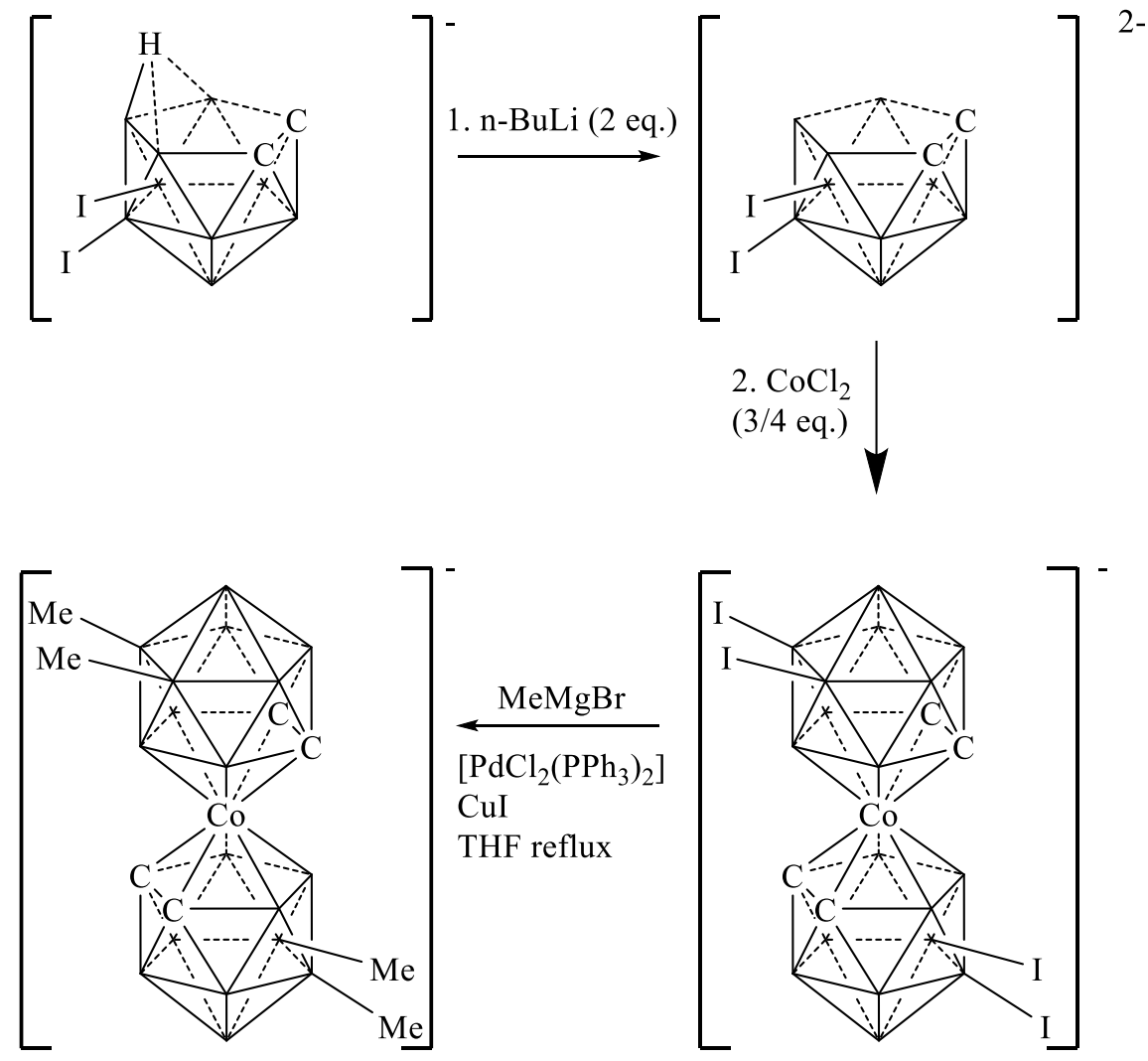
Table 1. $\mathrm{E}_{1 / 2}\left(\mathrm{Co}^{+}+2+\right)$ dependence upon the number and position of iodine substituent in platforms [1]-, [2]- and [3]-. $\mathrm{E}_{1 / 2}$ as well as $E_{L}(\mathrm{~L})$ are given in Volts.

\begin{tabular}{|c|c|c|c|c|}
\hline Compound & Substituents Position & $\begin{array}{l}\mathrm{E}_{1 / 2} v s . \\
\mathrm{F}_{\mathrm{c}+} / \mathrm{F}_{\mathrm{c}}\end{array}$ & $\begin{array}{l}\Delta \mathrm{E}_{1 / 2} \text { with } \\
\text { respect to } \\
\text { [1]- }\end{array}$ & $E_{L}(\mathbf{L})$ \\
\hline$[1]$ & - & $-1,80$ & - & 0.00 \\
\hline$[2]-$ & $\mathrm{C}_{\mathrm{c}}-\mathrm{Me}$ & $-1,72$ & +0.08 & +0.07 \\
\hline$[3]-$ & $\mathrm{C}_{c}-\mathrm{Ph}$ & $-1,66$ & +0.14 & +0.12 \\
\hline $\mathrm{I}_{4-[1]-}$ & $\mathrm{B}\left(9,9^{\prime}, 12,12^{\prime}\right)$ & $-1,15$ & +0.65 & +0.56 \\
\hline I4-[2]- & $\mathrm{B}\left(9,9^{\prime}, 12,12^{\prime}\right) ; \mathrm{C}\left(1,1^{\prime}\right)$ & $-1,03$ & +0.77 & +0.66 \\
\hline $\mathrm{I} 4-[3]-$ & $\mathrm{B}\left(9,9^{\prime}, 12,1^{\prime}\right) ; \mathrm{C}\left(1,1^{\prime}\right)$ & $-1,00$ & +0.80 & +0.69 \\
\hline $\mathrm{I} 8-[\mathbf{1}]-$ & $\mathrm{B}\left(8,8^{\prime}, 9,9^{\prime}, 10,10^{\prime}, 12,12^{\prime}\right)$ & $-0,68$ & +1.12 & +0.96 \\
\hline $\mathrm{I} 8-[2]-$ & $\mathrm{B}\left(8,8^{\prime}, 9,9^{\prime}, 10,10^{\prime}, 12,12^{\prime}\right) ; \mathrm{C}\left(1,1^{\prime}\right)$ & $-0,61$ & +1.19 & +1.02 \\
\hline I8-[3]- & $\mathrm{B}\left(8,8^{\prime}, 9,9^{\prime}, 10,10^{\prime}, 12,12^{\prime}\right) ; \mathrm{C}\left(1,1^{\prime}\right)$ & $-0,54$ & +1.26 & +1.09 \\
\hline
\end{tabular}


Table 2. $\mathrm{E}_{1 / 2}\left(\mathrm{Co}_{3+/ 2+}\right)$ dependence upon the number and position of iodine substituents in platform [1]-. $\mathrm{E}_{1 / 2}$ are given in Volts.

\begin{tabular}{|c|c|c|c|c|c|}
\hline Entry & Compound & Substituent' Position & $\begin{array}{l}\mathrm{E}_{1 / 2} v s . \\
F c+/ F c\end{array}$ & $\begin{array}{c}\Delta \mathrm{E}_{1 / 2} \text { with } \\
\text { respect to } \\
{[1]-}\end{array}$ & $E_{L}(\mathrm{~L})$ \\
\hline 1 & Me8-[1]- & $\mathrm{B}\left(8,8^{\prime}, 9,9^{\prime}, 10,10^{\prime}, 12,12^{\prime}\right)$ & $-1,92$ & $-0,12$ & -0.07 \\
\hline 2 & Me6-[1] & $\mathrm{B}\left(8,8^{\prime}, 9,9^{\prime}, 12,12^{\prime}\right)$ & $-1,89$ & $-0,09$ & -0.05 \\
\hline 3 & Me4-[1]- & $\mathrm{B}\left(9,9^{\prime}, 12,12^{\prime}\right)$ & $-1,84$ & $-0,04$ & -0.02 \\
\hline 4 & $\mathrm{Me}_{2}-[1]-$ & $\mathrm{B}\left(8,8^{\prime}\right)$ & $-1,82$ & $-0,02$ & -0.01 \\
\hline 5 & Me-[1]- & $\mathrm{B}(8)$ & $-1,81$ & $-0,01$ & 0.00 \\
\hline 6 & {$[1]-$} & - & $-1,80$ & - & 0.00 \\
\hline 7 & $\mathrm{I}-[1]-$ & $\mathrm{B}(8)$ & $-1,50$ & $+\mathbf{0 , 3 0}$ & +0.18 \\
\hline 8 & $\mathrm{I}_{2}-[1]-$ & $\mathrm{B}\left(9,9^{\prime}\right)$ & $-1,46$ & $+0,34$ & +0.29 \\
\hline 9 & $\mathrm{I}_{2}-[1]-$ & $\mathrm{B}\left(8,8^{\prime}\right)$ & $-1,32$ & $+0,48$ & +0.41 \\
\hline 10 & $\mathrm{I}_{2}-[1]-$ & $\mathrm{B}\left(4,4^{\prime}\right)$ & $-1,24$ & $+0,56$ & +0.48 \\
\hline 11 & $\mathrm{I} 4-[\mathbf{1}]-$ & $\mathrm{B}\left(9,9^{\prime}, 12,1^{\prime}\right)$ & $-1,15$ & $+0,65$ & +0.56 \\
\hline 12 & I4-[1]- & $\mathrm{B}\left(4,4^{\prime}, 7,7^{\prime}\right)$ & $-0,77$ & $+\mathbf{1 , 0 3}$ & +0.89 \\
\hline 13 & I6-[1]- & $\mathrm{B}\left(8,8^{\prime}, 9,9^{\prime}, 12,12^{\prime}\right)$ & $-0,82$ & $+\mathbf{0 , 9 8}$ & +0.84 \\
\hline 14 & I6-[1]- & $\mathrm{B}\left(4,4^{\prime}, 9,9^{\prime}, 12,12^{\prime}\right)$ & $-0,80$ & $+1,00$ & +0.86 \\
\hline 15 & $\mathrm{I} 8-[1]-$ & $\mathrm{B}\left(8,8^{\prime}, 9,9^{\prime}, 10,10^{\prime}, 12,12^{\prime}\right)$ & $-0,68$ & $+1,12$ & $+\mathbf{0 . 9 7}$ \\
\hline
\end{tabular}


Table 3. B3LYP calculated free energies (in a.u.) for the three optimized isomers ( $a, b$ and c in Figure 3) for the fifteen $\mathrm{Co} 2+$ and $\mathrm{Co}_{3}+$ compounds 1-15. The values in parenthesis correspond to the free energy difference between the isomers (in $\mathrm{kcal} / \mathrm{mol}$ ) taking as reference the usually most stable c isomer. Only for the compound 12, the c isomer is significantly less stable than the other two isomers due to the simultaneous $4,4^{\prime}, 7,7^{\prime}$ substitution on each $\mathrm{C}_{2} \mathrm{~B}_{3}$ face.

\begin{tabular}{rrrrrrrrrrrrr}
\hline & \multicolumn{1}{c}{$\mathbf{C o 2}+\mathbf{a}$} & \multicolumn{2}{c}{$\mathbf{C o 2}+\mathbf{b}$} & $\mathbf{C 0 2}+\mathbf{c}$ & \multicolumn{2}{c}{ Co3+ } & \multicolumn{2}{c}{ Co3+ b } & Co3+ \\
\hline $\mathbf{1}$ & -1072.2653 & $(4.3)$ & -1072.2708 & $(0.8)$ & -1072.2721 & -1072.2897 & $(4.7)$ & -1072.2976 & $(-0.3)$ & -1072.2972 \\
$\mathbf{2}$ & -993.6799 & $(5.0)$ & -993.6867 & $(0.8)$ & -993.6879 & -993.7010 & $(6.3)$ & -993.7093 & $(1.1)$ & -993.7111 \\
$\mathbf{3}$ & -915.1049 & $(2.6)$ & -915.1065 & $(1.6)$ & -915.1091 & -915.1257 & $(2.7)$ & -915.1301 & $(0.0)$ & -915.1300 \\
$\mathbf{4}$ & -836.5090 & $(5.0)$ & -836.5137 & $(2.0)$ & -836.5169 & -836.5270 & $(5.6)$ & -836.5349 & $(0.7)$ & -836.5360 \\
$\mathbf{5}$ & -797.2175 & $(5.4)$ & -797.2238 & $(1.5)$ & -797.2261 & -797.2398 & $(3.6)$ & -797.2435 & $(1.3)$ & -797.2455 \\
$\mathbf{6}$ & -757.9246 & $(6.9)$ & -757.9327 & $(1.7)$ & -757.9355 & -757.9485 & $(3.7)$ & -757.9523 & $(1.3)$ & -757.9544 \\
$\mathbf{7}$ & -768.7472 & $(20.8)$ & -768.7798 & $(0.3)$ & -768.7803 & -768.7722 & $(8.6)$ & -768.7808 & $(3.3)$ & -768.7860 \\
$\mathbf{8}$ & -779.6126 & $(5.6)$ & -779.6174 & $(2.6)$ & -779.6216 & -779.6103 & $(4.5)$ & -779.6151 & $(1.5)$ & -779.6174 \\
$\mathbf{9}$ & -779.5996 & $(14.8)$ & -779.6112 & $(7.5)$ & -779.6232 & -779.5915 & $(15.1)$ & -779.6082 & $(4.6)$ & -779.6155 \\
$\mathbf{1 0}$ & -779.5908 & $(5.9)$ & -779.5938 & $(4.0)$ & -779.6001 & -779.5855 & $(4.9)$ & -779.5846 & $(5.4)$ & -779.5932 \\
$\mathbf{1 1}$ & -801.2783 & $(10.6)$ & -801.2912 & $(2.5)$ & -801.2952 & -801.2649 & $(5.2)$ & -801.2709 & $(1.4)$ & -801.2731 \\
$\mathbf{1 2}$ & -801.2415 & $(-6.5)$ & -801.2575 & $16.5)$ & -801.2311 & -801.2161 & $(-5.3)$ & -801.2296 & $(-13.7)$ & -801.2076 \\
$\mathbf{1 3}$ & -822.9299 & $(17.0)$ & -822.9443 & $(8.0)$ & -822.9570 & -822.8902 & $(17.9)$ & -822.9101 & $(5.4)$ & -822.9187 \\
$\mathbf{1 4}$ & -822.9267 & $(8.8)$ & -822.9332 & $(4.7)$ & -822.9406 & -822.8903 & $(5.5)$ & -822.8909 & $(5.1)$ & -822.8991 \\
$\mathbf{1 5}$ & -844.5851 & $(16.5)$ & -844.5996 & $(7.4)$ & -844.6113 & -844.5352 & $(18.4)$ & -844.5552 & $(5.9)$ & -844.5646 \\
\hline
\end{tabular}


Figure 1. Cobaltabisdicarbollide-based electroactive frameworks.
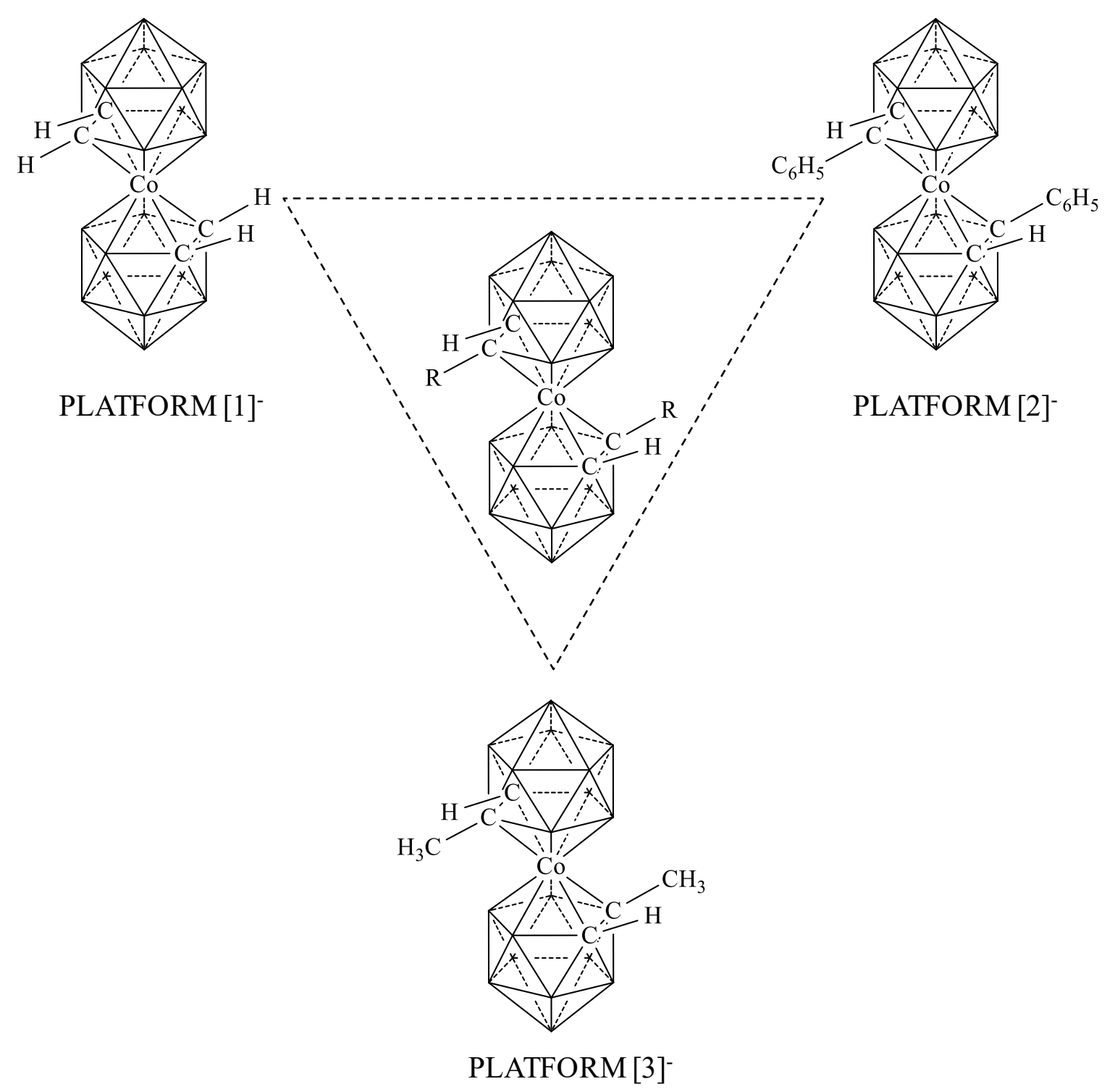
Figure 2. Rough approximation of the front view of the icosahedron cluster in a hexagonal representation.

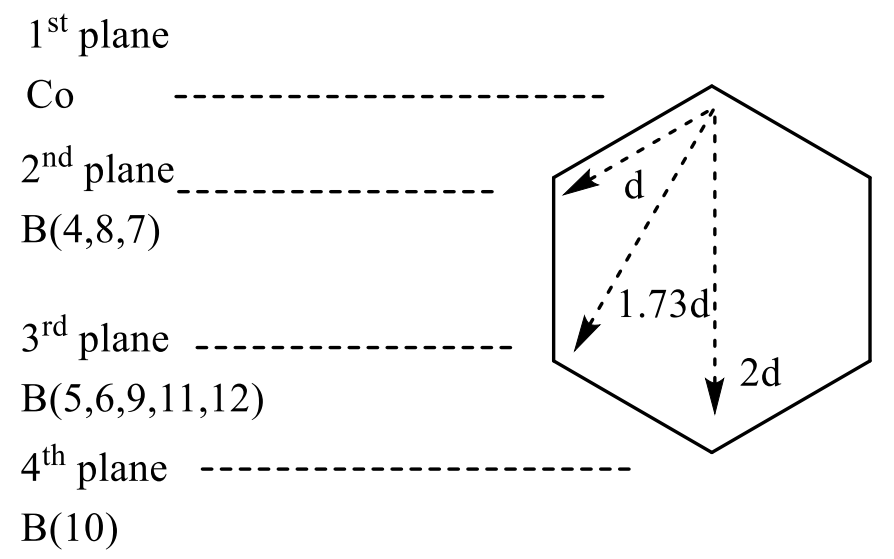


Figure 3.- Model structures of the three isomers studied for the fifteen complexes 1-15.

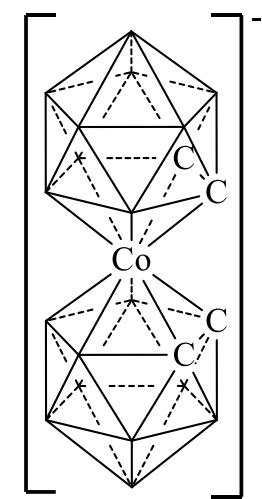

Isomer a
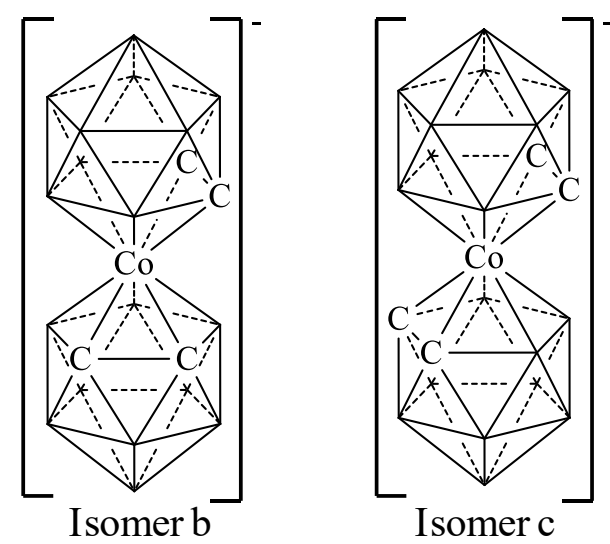
Figure 4. (a) Dependence of the experimental redox potential for cobaltabisdicarbollide derivatives (1-15 entries in Table 2) with the DFT calculated values (black circles) and the HOMO energies corresponding to the solvated molecule (white circles). (b) Representation of the HOMO orbitals corresponding to Mes-[1]-, [1]- and I8-[1]- (entries 1, 6 and 15, respectively, in Table 2).

(a)

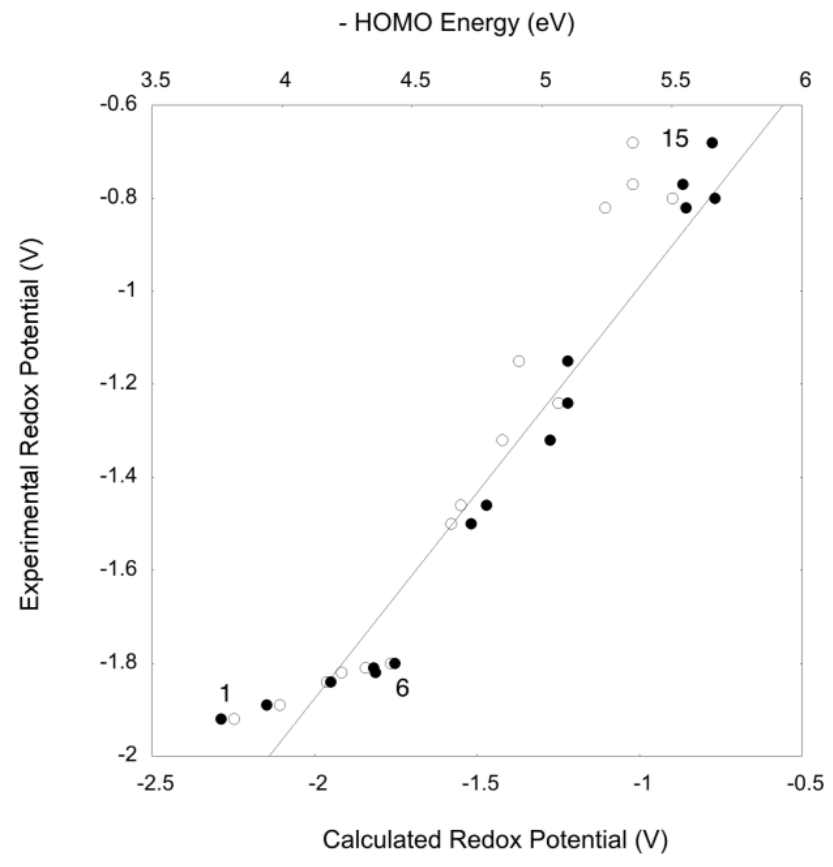

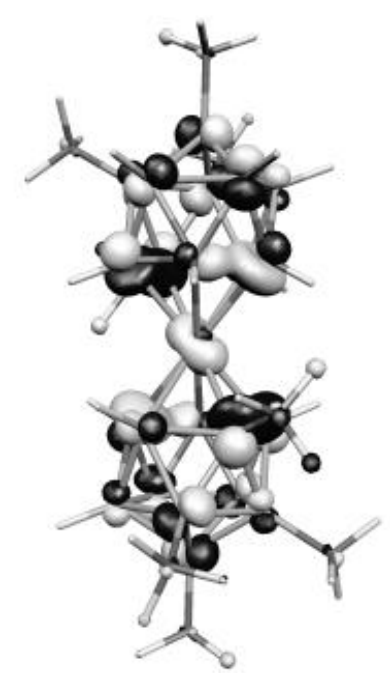

(b)

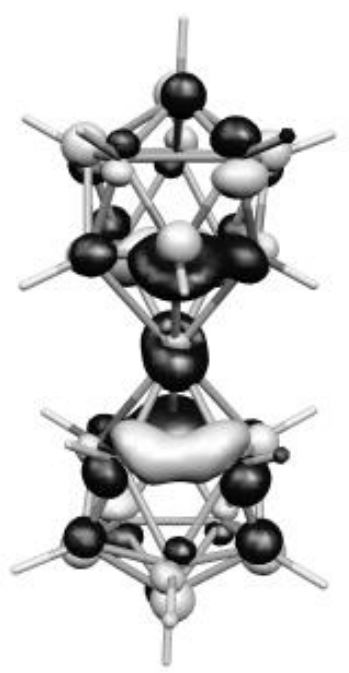

[1]-

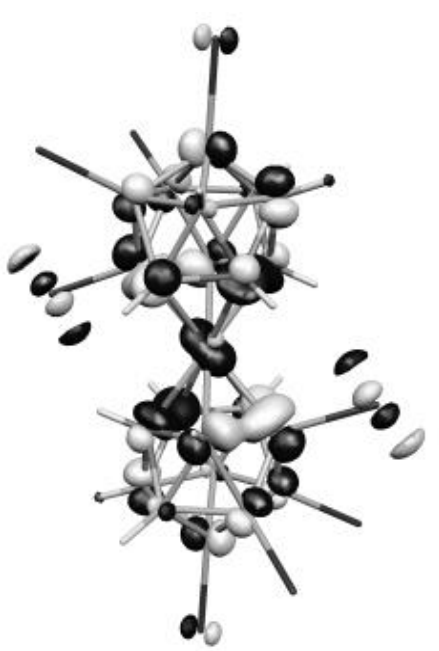

I8-[1]- 
Figure 5. ${ }_{11} \mathrm{~B}\{1 \mathrm{H}\}-\mathrm{NMR}$ of the reduced form $\left[3,3^{\prime}-\mathrm{Co}\left(8,9,10,12-\mathrm{I}_{4}-1,2-\text { closo- } \mathrm{C}_{2} \mathrm{~B} 9 \mathrm{H}_{7}\right)_{2}\right]_{2-}$ (I8-[1] $\left.2_{2-}\right)$ in black $v s$ the oxidized one $\left[3,3^{\prime}-\mathrm{Co}\left(8,9,10,12-\mathrm{I}_{4}-1,2-\right.\right.$ closo- $\left.\left.\mathrm{C}_{2} \mathrm{~B} 9 \mathrm{H}_{7}\right) 2\right]-\left(\mathrm{I} 8-[1]_{-}\right)$in red.

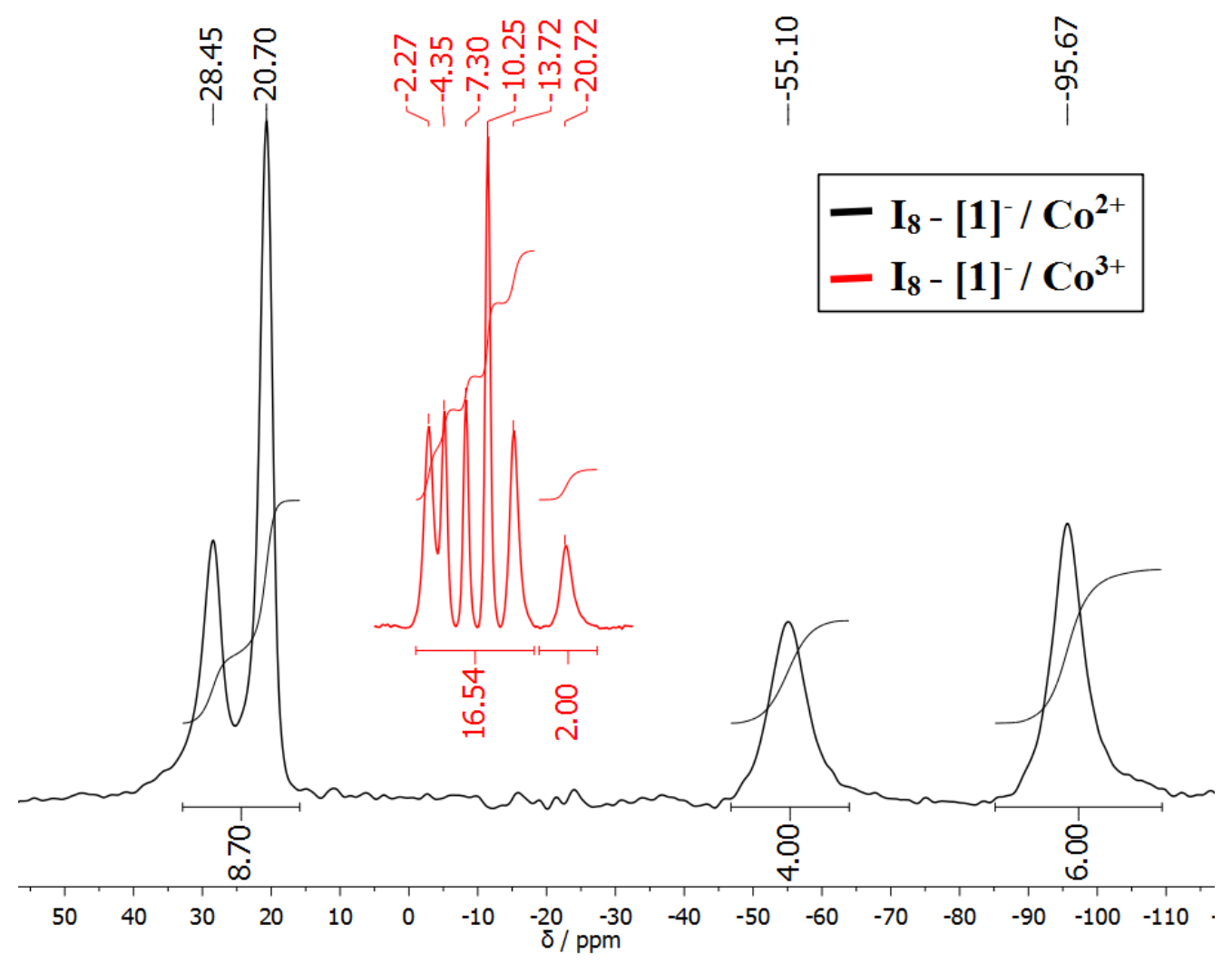


Figure 6.- Electron paramagnetic resonance spectra (EPR) of I8-[1] $]_{2-}$

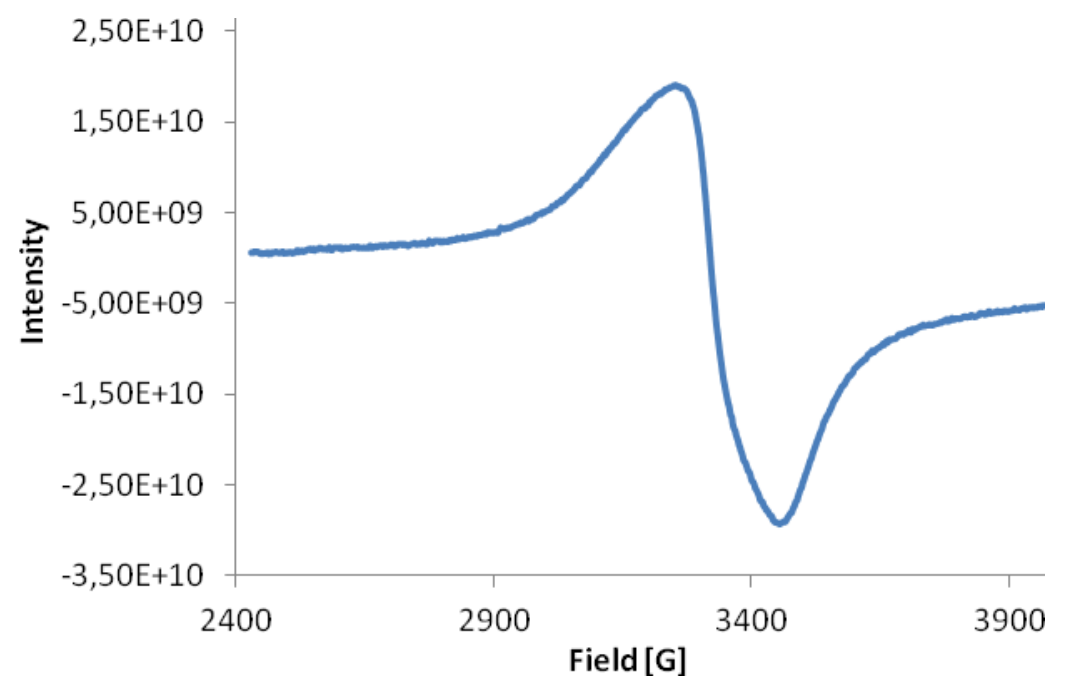


Figure 7. Air stability of both compounds in the couple $\left[3,3^{\prime}-\mathrm{Co}(8,9,10,12-\mathrm{I} 4-1,2-\right.$ closo$\left.\left.\mathrm{C}_{2} \mathrm{~B} 9 \mathrm{H} 7\right)_{2}\right]-1 / 2$.

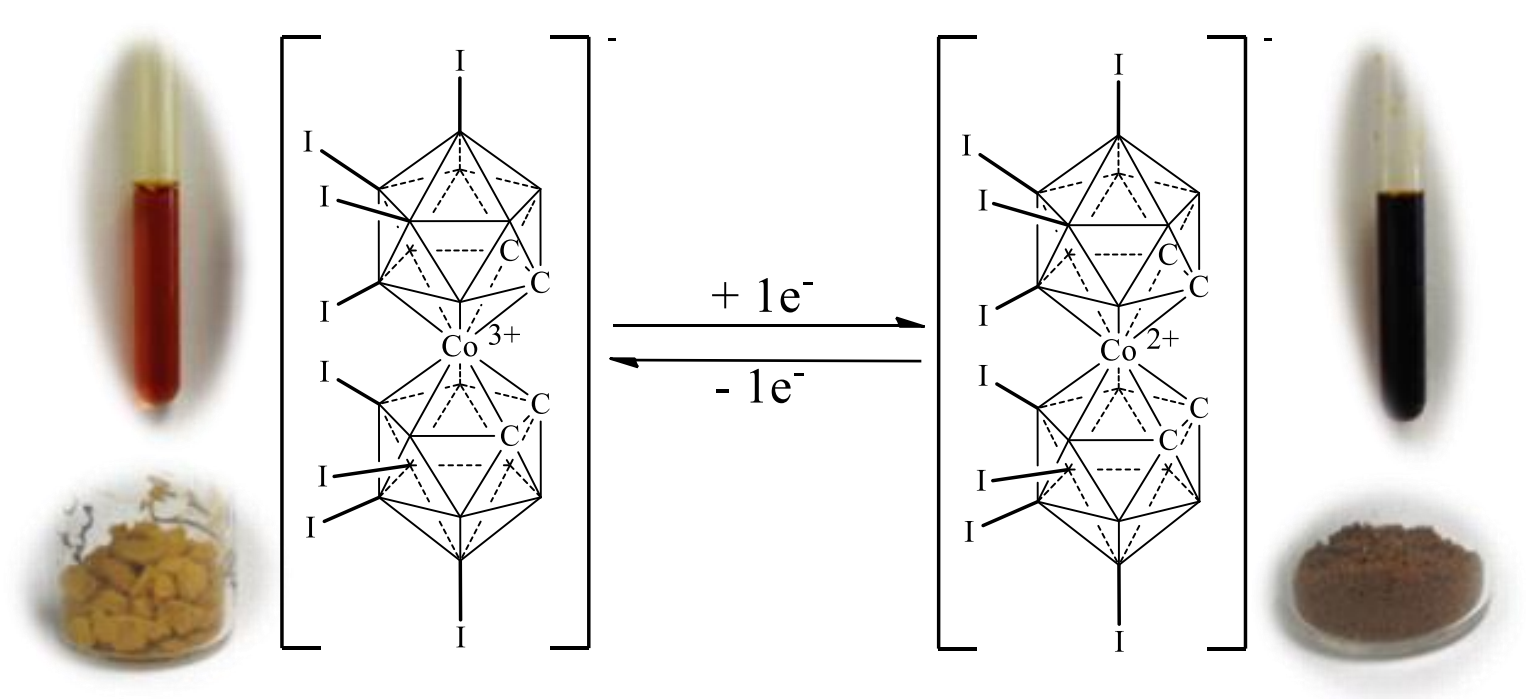




\section{References}

[1] N. N. Greenwood, A. Earnshaw, in Chemistry of the Elements 2nd edn, Elsevier, 1997.

[2] P. Farràs, E. J. Juárez-Pérez, M. Lepsik, F. Luque, R. Núñez, F. Teixidor, Chem. Soc. Rev. 2012, 41, 3445-3463.

[3] I. B. Sivaev, V. I. Bregadze, Collect. Czeck. Chem. Commun. 1999, 64, 783-805.

[4] D. Olid, R. Núñez, C. Viñas, F. Teixidor, Chem. Soc. Rev. 2013, 42, 3318-3336.

[5] M. Corsini, F. Fabrizi de Biani, P. Zanello, Coord. Chem. Rev. 2006, 250, 1351-1372.

[6] J. Plesek, Chem. Rev. 1992, 92 (2), 269-278.

[7] F. Teixidor, C. Viñas, Pure Appl. Chem. 2012, 84, 2457-2465.

[8] a) O. N. Kazheva, G. G. Alexandrov, A. V. Kravchenko, V. A. Starodub, I. A. Lobanova, I. B. Sivaev, V. I. Bregadze, L. V. Titov, L. I. Buravov, O. A. Dyachenko, J. Organomet. Chem. 2009, 694, 2336-2342; b) L. I. Zakharkin, V. A. Olshevskaya, E. V. Balagurova, P. V. Petrovskii, Russ. J. Gen. Chem. 2000, 70, 550-551; c) O. N. Kazheva, G. G. Aleksandrov, A. V. Kravchenko, V. A. Starodub, G. G. Zhigareva, I. B. Sivaev, V. I. Bregadze, L. I. Buravov, L. V. Titov, O. A. Dyachenko, Russ. Chem. Bull. 2010, 59, 1137-1144.

[9] a) P. K. Hurlburt, R.L. Miller, K.D. Abney, T.M. Foreman, R.J. Butcher, S.A. Kinkhead, Inorg. Chem. 1995, 34, 5215-5219; b) L. Matel, F. Macasek, P. Rajec, S. Hermanek, J. Plesek, Polyhedron 1982, 1, 511-519; c) P. Selucky, K. Base, J. Plesek, S. Hermanek, J. Rais, Chem. Abstr. 1986, 104, 1866379g

[10] P. González-Cardoso, A. I. Stoica, P. Farràs, A. Pepiol, C. Viñas, F. Teixidor, Chem. Eur. J. 2010, 16, 6660-6665. 
[11] A. Pepiol, F. Teixidor, R. Sillanpää, M. Lupu, C. Viñas, Angew. Chem. Int. Ed. 2011, $50,12491-12495$.

[12] A. A. Popov, I. E. Kareev, N. B. Shustova, E. B. Stukalin, S. F. Lebedkin, K. Seppelt, S. H. Strauss, O. V. Boltalina, L. Dunsch, J. Am. Chem. Soc. 2007, 129, 11551-11568.

[13] L. Pauling, J. Am. Chem. Soc. 1932, 54, 3570-3582.

[14] The polarization of exo-cluster bonds in carborane type architectures is not always intuitive. For a recent example and discussion of this situation, see A. El-Hellani, C. E. Kefalidis, F. S. Tham, L. Maron, V. Lavallo, Organometallics 2013, 32, 6887-6890.

[15] T. Daeneke, A. J. Mozer, T.-H. Kwon, N. W. Duffy, A. B. Holmes, U. Bach, L. Spiccia, Energy Environ. Sci. 2012, 5, 7090-7099.

[16] M. D. Mortimer, C. B. Knobler, M. F. Hawthorne, Inorg. Chem. 1996, 35, 5750-5751.

[17] A. J. L. Pombeiro, Eur. J. Inorg. Chem. 2007, 1473-1482.

[18] A. J. L. Pombeiro, in: Inorganic Electrochemistry (Eds.: F. Scholz, C. J. Pickett), Encyclopedia of Electrochemistry, Wiley-VCH, Weinheim, 2006, vol. 7A, p.77-108.

[19] C. J. Pickett, in: G. Wilkinson, R. D. Gillard, J. A. McCleverty (Eds.), Comprehensive Coordination Chemistry, Pergamon Press, Oxford, 1987, vol. 1, chapter 8.3, p. 493.

[20] a) A. B. P. Lever, Inorg. Chem. 1990, 29, 1271-1285; b) A. B. P. Lever, Inorg. Chem. 1991, 30, 1980-1985; c) Lever, A.B.P.; Dodsworth, E.S. Inorganic Electronic Structure and Spectroscopy; Solomon, E.I, Lever, A.B.P, Eds.; Wiley: New York, 1999; vol. 2, pp. 227-287; c) A. B. P. Lever, in: Comprehensive Coordination Chemistry II, vol. 2 (Ed.: A. B. P. Lever), Elsevier, Oxford, 2004, chapter 2.19, p. 251.

[21] B. E. Bursten, J. Am. Chem. Soc. 1982, 104, 1299-1304. 
[22] For metal-centered redox reactions the equation: $E_{\text {calc }}\left(\mathrm{M}_{\mathrm{n}+1} / \mathrm{M}_{\mathrm{n}}\right)=S_{\mathrm{M}} \Sigma E_{L}(\mathrm{~L})+I_{\mathrm{M}}$ is applied. The parameters $S_{\mathrm{M}}$ and $I_{\mathrm{M}}$ for a particular $\mathrm{Mn}_{\mathrm{n}+1 / \mathrm{n}}$ couple are constants. In organic solvents, for $\mathrm{Co}_{3}+2+$ are $0.83(0.05)$ and $-1.16(0.13) \mathrm{V}$, refereed to SHE.

[23] P. Farràs, F. Teixidor, I. Rojo, R. Kivekäs, R. Sillanpää, P. González-Cardoso, C.

Viñas, J. Am. Chem. Soc. 2011, 133, 16537-16552.

[24] Calculations were performed using the Gaussian09 (revisión D01) with the hybrid B3LYP functional together with LANL2DZ basis set (Hay-Wadt effective core potentials for the iodine and cobalt atoms).

[25] a) W. Kaim, N. S. Hosmane, S. Zalis, J. A. Maguire, W. N. Lipscomb, Angew. Chem. Int. Ed. 2009, 48, 5082-5091; b) J. Kahlert, H.-G. Stammler, B. Neumann, R. A. Harder, L. Weber, M. A. Fox, Angew. Chem. Int. Ed. 2014, 53, 3702-3705; c) K. Vyakaranam, Z. Havlas, J. Michl, J. Am. Chem. Soc. 2007, 129, 4172-4174; d) M. Asay, C. E. Kefalidis, J. Estrada, D. S. Weinberger, J. Wright, C. E. Moore, A. L. Rheingold, L. Maron, V. Lavallo, Angew. Chem. Int. Ed. 2013, 52, 11560-11563.

[26] M. M. Fein, D. Grafstein, J. E. Paustian; J. Bobinski, B. M. Lischtein, N. Mayes, N. N. Schwartz, M. S. Cohen, Inorg. Chem. 1963, 2, 1115-1119.

[27] T. L. Heying, J. W. Ager, Jr., S. L. Clark, D. J. Mangold, H. L. Goldstein, M. Hillman, R. J. Polak, J. W. Szymanski, Inorg. Chem. 1963, 2, 1089-1092.

[28] G. Barberà, A. Vaca, F. Teixidor, R. Sillanpää, R. Kivekäs, C. Viñas, Inorg. Chem. 2008, 47, 7309-7316.

[29] F.P. Olsen, M.F. Hawthorne, Inorg. Chem. 1965, 4, 1839-1840.

[30] R.H. Pak, R.R.Kane, C.B. Knobler, M.F. Hawthorne, Inorg. Chem. 1994, 33, 53555357. 
[31] I. Rojo, F. Teixidor, R. Kivekäs, R. Sillanpää, C. Vinas, Organometallics 2003, 22, $4642-4646$.

[32] Gaussian 09 (Revision D01): M. J. Frisch, G. W. Trucks, H. B. Schlegel, G. E. Scuseria, M. A. Robb, J. R. Cheeseman, G. Scalmani, V. Barone, B. Mennucci, G. A. Petersson, H. Nakatsuji, M. Caricato, X. Li, H. P. Hratchian, A. F. Zmaylov, I. J. Bloino, G. Zheng, J. L. Sonnenberg, M. Hada, M. Ehara, K. Toyota, R. Fukuda, J. Hasegawa, M. Ishida, T. Nakajima, Y. Honda, O. Kitao, H. Nakai, T. Vreven, J. J. A. Montgomery, J. E. Peralta, F. Ogliaro, M. Bearpark, J. J. Heyd, E. Brothers, K. N. Kudin, V. N. Staroverov, R. Kobayashi, J. Normand, K. Raghavachari, A. Rendell, J. C. Burant, S. S. Iyengar, J. Tomasi, M. Cossi, N. Rega, J. M. Millam, M. Klene, J. E. Knox, J. B. Cross, V. Bakken, C. Adamo, J. Jaramillo, R. Gomperts, R. E. Stratmann, O. Yazyev, A. J. Austin, C. Cammi, J.W. Pomelli, R. Ochterski, R. L. Martin, K. Morokuma, V. G. Zakrzewski, G. A. Voth, P. Salvador, J. J. Dannenberg, S. Dapprich, A. D. Daniels, O. Farkas, J. B. Foresman, J.V. Ortiz, J. Cioslowski and D. J. Fox,Wallingford, CT, 2009.

[33] A. D. Becke, J. Chem. Phys. 1993, 98, 5648-5652.

[34] P. J. Hay, W. R. Wadt, J. Chem. Phys. 1985, 82, 270-283.

[35] M.-H. Baik, R. A. Friesner, J. Phys. Chem. A 1985, 82, 7407-7412. 